\title{
Movie genome: alleviating new item cold start in movie recommendation
}

\author{
Yashar Deldjoo, et al. [full author details at the end of the article]
}

Received: 23 December 2017 / Accepted in revised form: 19 January 2019 / Published online: 26 February 2019 (c) The Author(s) 2019

\begin{abstract}
As of today, most movie recommendation services base their recommendations on collaborative filtering $(\mathrm{CF})$ and/or content-based filtering (CBF) models that use metadata (e.g., genre or cast). In most video-on-demand and streaming services, however, new movies and TV series are continuously added. CF models are unable to make predictions in such a scenario, since the newly added videos lack interactions-a problem technically known as new item cold start (CS). Currently, the most common approach to this problem is to switch to a purely CBF method, usually by exploiting textual metadata. This approach is known to have lower accuracy than CF because it ignores useful collaborative information and relies on human-generated textual metadata, which are expensive to collect and often prone to errors. User-generated content, such as tags, can also be rare or absent in CS situations. In this paper, we introduce a new movie recommender system that addresses the new item problem in the movie domain by (i) integrating state-of-the-art audio and visual descriptors, which can be automatically extracted from video content and constitute what we call the movie genome; (ii) exploiting an effective data fusion method named canonical correlation analysis, which was successfully tested in our previous works Deldjoo et al. (in: International Conference on Electronic Commerce and Web Technologies. Springer, Berlin, pp 34-45, 2016b; Proceedings of the Twelfth ACM Conference on Recommender Systems. ACM, 2018b), to better exploit complementary information between different modalities; (iii) proposing a two-step hybrid approach which trains a CF model on warm items (items with interactions) and leverages the learned model on the movie genome to recommend cold items (items without interactions). Experimental validation is carried out using a system-centric study on a large-scale, real-world movie recommendation dataset both in an absolute cold start and in a cold to warm transition; and a user-centric online experiment measuring different subjective aspects, such as satisfaction and diversity. Results show the benefits of this approach compared to existing approaches.
\end{abstract}

Markus Schedl

markus.schedl@jku.at

Extended author information available on the last page of the article 
Keywords Movie recommender systems · Cold start · Warm start · Semi-cold start . New item · Multimedia features · Content-based · Audio descriptors · Visual descriptors · Multimodal fusion · Hybrid recommender system · Feature weighting · Collaborative-enriched content-based filtering $\cdot$ Canonical correlations analysis

\section{Introduction}

A dramatic rise in the generation of video content has occurred in recent years. According to Cisco, the largest networking company across the globe, by 2020 more than $75 \%$ of the world's mobile data traffic will be video, or even $80 \%$ when video and audio data are considered together (Cisco visual networking index 2016). This rise has been fueled by online social network users who upload/post a staggering amount of user-generated video on a daily basis. For instance, as of 2018, YouTube ${ }^{1}$ users upload over $400 \mathrm{~h}$ of video every minute. This translates to about 3 years of non-stop watching in order to consume all videos uploaded to YouTube in a single hour. Similarly, Instagram ${ }^{2}$ users post nearly 70 million photos and videos each day (Xu et al. 2017).

In this context, video recommender systems play an important role in helping users of online streaming services, as well as of social networks, cope with this rapidly increasing volume of videos and provide them with personalized experiences. Nevertheless, the growing availability of digital videos has not been fully accompanied by comfort in their accessibility via video recommender systems. The causes of this problem are twofold: (i) the type of recommendation models in service today, which are heavily dependent on usage data (in particular, implicit or explicit preference feedback) and/or metadata (e.g., genre and cast associated with the videos) (cf. Sect. 1.1), and (ii) the nature of video data, which are information intensive when compared to other media types, such as music or images (cf. Sect. 1.2). In the following article, we analyze each of these dimensions. Throughout this paper, we will use a number of abbreviations, which, for convenience are summarized in Table 1.

\subsection{New item cold-start recommendation in the movie domain}

To date, collaborative filtering (CF) methods (Koren and Bell 2015) lie at the core of most real-word movie recommendation engines, due to their state-of-the-art accuracy (McFee et al. 2012; Yuan et al. 2016). In most video-streaming services, however, new movies and TV series are continuously added. CF models are not capable of providing meaningful recommendations when items in the catalogue contain few interactions, a problem commonly known as the cold start (CS) problem. The most severe case of CS is when new items are added that lack any interactions, technically known as the new item CS problem. ${ }^{3}$ In such a situation, CF models are completely unable to make predictions. As such, these new items are not recommended, go unnoticed by a large

\footnotetext{
1 https://www.youtube.com/.

2 https://www.instagram.com/.

3 Note that videos without interactions can also be old videos that have been never been watched by a user.
} 
Table 1 List of abbreviations used throughout the paper

\begin{tabular}{ll}
\hline Abbreviation & Term \\
\hline MM & Multimedia \\
RS & Recommender systems \\
VRS & Video recommender systems \\
MRS & Movie recommender systems \\
MMRS & Multimedia recommender systems \\
CS & Cold start \\
WS & Warm start \\
AVF & Aesthetic visual features \\
BLF & Block-level features \\
CBF & Content-based filtering \\
CF & Collaborative filtering \\
CF-MMRS & Collaborative filtering multimedia recommender system \\
CB-MMRS & Content-based multimedia recommender system \\
CA-MMRS & Context-aware multimedia recommender system \\
BPR & Bayesian personalized ranking \\
KNN & K-Nearest Neighbor \\
CFeCBF & Collaborative-filtering enriched content-based filtering \\
\hline
\end{tabular}

part of the user community, and remain unrated, creating a vicious circle in which a set of items in the RS is left out of the vote/recommendation process (Bobadilla et al. 2012). Being able to provide high-quality recommendations for cold items has several advantages. Firstly, it will increase the novelty of the recommendations, which is a highly desirable property and inherent in the user-centric and business-centric goals of RS, i.e., the discovery of new content and the increase of revenues (Aggarwal 2016b; Liu et al. 2014). Secondly, providing good new movie recommendations will allow enough interactions/feedbacks to be collected in a brief amount of time enabling effective CF recommendation. Despite previous efforts, the new item CS problem remains far from being solved in the general case, and most existing approaches suffer from it (Bobadilla et al. 2012; Zhou et al. 2011; Zhang et al. 2011).

Currently, the most common approach to counteracting the new item CS problem is to switch to a pure CBF (de Gemmis et al. 2015; Lops et al. 2011) method by using additional attribute content for items, usually by resorting to metadata provided in textual form (Liu et al. 2011). This approach is known to have lower accuracy than CF because it ignores potentially useful collaborative information and typically relies on human-generated textual metadata, which are often noisy, expensive to collect, and sparse. More importantly, extra information for cold items is not always available on the web (especially in user-generated form), even if it is available in abundance for warm items (Zhang et al. 2015). In addition, given the unstructured or semi-structured nature of metadata, they often require complex natural language processing (NLP) techniques for pre-processing, e.g., syntactic and semantic analysis or topic modeling (Aggarwal 2016a). 
Many approaches have been proposed to address the new item CS issue, mainly based on hybrid CF and CBF models (Lika et al. 2014; Cella et al. 2017; Sharma et al. 2015; Ferrari Dacrema et al. 2018). Most recent work relies on machine learning to combine content and collaborative data. We focus on feature weighting rather than on other types of hybrids (e.g., joint matrix factorization) because we aim to build a hybridization strategy that can be easily applied to a CBF model. For instance, the authors in Gantner et al. (2010) proposed a method to map item features into the item embeddings learned in a matrix factorization algorithm, while the authors in Schein et al. (2002) defined a probabilistic model trained via expectation minimization. Another example is Sharma et al. (2015), where the authors proposed a feature weighting model that learns feature weight by optimizing the ranking of the recommendations over the user interactions for warm items.

Addressing this issue, the main contribution of the present work is to improve the current state of the art by presenting a generalized, two-step machine learning approach to feature weighting and by testing its effectiveness on both editorial features and stateof-the-art multimedia (MM) descriptors. Hereafter, for simplicity, we refer to items without interactions as cold items and items containing interactions as warm items.

\subsection{Video as an information-intensive multimodal media type}

When we watch a movie, we can effortlessly register many details conveyed to us through different multimedia channels - in particular, the audio and visual channels. As a result, the perception of a film in the eyes of viewers is influenced by many factors related not only related to, e.g., the genre, cast, and plot, but also according to the overall film style (Bordwell et al. 1997). These factors affect the viewer's experience. For example, two movies may be from the same genre and director, but they can be different based on the movie style. Consider as an example Empire of the Sun and Schindler's List, both dramatic movies directed by Steven Spielberg and both describing historical events. However, they are completely different in style, with Schindler's List shot like a documentary in black and white, while Empire of the Sun is shot using bright colors and makes heavy use of special effects. Although these two movies are similar with respect to traditional metadata (e.g., director, genre, year of production), their different styles are likely to affect the viewers' feelings and opinions differently (Deldjoo et al. 2016d). In fact, the film story is first created by the author and the comprehension of the cinematographical language by the spectator reshapes the story (Fatemi and Mulhem 1999). The notion of story in a movie depends on semantic content (reflected better in metadata) reshaped through stylistic cinematography elements (reflected better in multimedia content). These discernible characteristics of movie content meet users' different information needs.

The extent to which content-based approaches are used, and even the way "content" is interpreted, varies between domains. While extracting descriptive item features from text, audio, image, and video content is a well-established research domain in the multimedia community (Lew et al. 2006), the recommender system community has long considered metadata, such as the title, genre, tags, actors, or plot of a movie, as the single source for content-based recommendation models, thereby disregarding 
the wealth of information encoded in the actual content signals. In order for MRS to make progress in recommending the right movies to the right user(s), they need to be able to interpret such multimodal signals as an ensemble and utilize item models that take into account the maximum possible amount of this information. We refer to such a holistic description of a movie, taking into account all available modalities, as its movie genome, since it can be considered the footprint of both content and style (Bronstein et al. 2010; Jalili et al. 2018). ${ }^{4}$

In this paper, we specifically address the above-mentioned shortcomings of purely metadata-based MRS by proposing a practical solution for the new item CS challenge that exploits the movie genome. We set out to answer the following research questions:

RQ1 Can the exploitation of movie genome describing rich item information as a whole, provide better recommendation quality compared with traditional approaches that use editorial metadata such as genre and cast in CS scenarios?

RQ2 Which visual and audio information better captures users' movie preferences in CS scenarios?

RQ3 Can we effectively leverage past user behavior data on warm items (items with interactions) to enrich the overall item representation and improve our ability to recommend cold items when interactions are not available?

The remainder of this article is structured as follows. Section 2 positions our work in the context of the state of the art and highlights its novel contributions. Section 3 introduces the proposed general content-based recommendation framework. Sections 4 and 5 report on the experimental validation, namely the experimental setup and parameter tuning, offline experimentation, and a user study in a web survey, respectively. Section 6 concludes the article in the context of the research questions and discusses limitations and future perspectives.

\section{State of the art}

One main contribution of this work is the introduction of a solution for the new item CS problem in the multimodal movie domain. In this section, we therefore review the existing, state-of-the-art approaches in content-based multimedia recommender systems (Sect. 2.1) and feature weighting for CS recommender systems (Sect. 2.2) and position our contribution (Sect. 2.3).

\subsection{Content-based multimedia recommendation}

A multimedia recommendation system is a system that recommends a particular media type, such as audio, image, video, and/or text, to the users (Deldjoo et al. 2018e,f). We therefore organize the state-of-the-art CB-MMRS based on the target media type, namely: (i) audio recommendation, (ii) image recommendation, and (iii) video recommendation. In the following subsections, we describe each of these systems.

\footnotetext{
4 Similar to biological DNA composed of long sequences of four letters A, T, C, G referred to as nucleotides.
} 


\subsubsection{Audio recommendation}

The most common example of audio recommendation is music recommendation (Schedl et al.2018; Vall et al. 2019). Over the past several years, a wealth of approaches, including $\mathrm{CF}, \mathrm{CBF}$, context-aware recommenders, and hybrid methods, have been proposed to address this task. An overview of popular approaches can be found in Schedl et al. (2015, 2018). Perhaps more than in other MM domains, CB recommenders have attracted substantial interest from researchers in the music domain, not least due to their superior performance in CS scenarios.

Recent work has proposed deep learning-based CB approaches. For instance, the authors in van den Oord et al. (2013) use a deep convolutional neural network (CNN) trained on audio features, more precisely, on the log-scaled Mel spectrograms extracted from 3-second-snippets of the audio, resulting in a latent factor representation for each song. The authors evaluate their approach for tag prediction and music recommendation using the Million Song Dataset (Bertin-Mahieux et al. 2011). In tenfold cross-validation experiments using 50-dimensional latent factors, they show that the $\mathrm{CNN}$ outperforms both metric learning to rank and a multilayer perceptron trained on bag-of-words representations of vector-quantized Mel frequency cepstral coefficients (MFCC) (Logan 2000a) in both tasks.

In contrast to such automatic feature learning approaches, some systems use human-made annotations of music. Perhaps, the most notable and well-known is the proprietary Music Genome Project (MGP), ${ }^{5}$ which is used by music streaming major Pandora. ${ }^{6}$ MGP captures various attributes of music and uses them in a CBF recommender system. These attributes are created by musical experts who manually annotate songs. Pandora uses up to 450 specific descriptors per song, such as "aggressive female vocalist", "prominent backup vocals", or "use of unusual harmonies".

In our approach, we follow a strategy in between these two extremes (i.e., fully automated feature learning by deep learning and pure manual expert annotations). The proposed movie genome uses well-established, state-of-the-art audio descriptors that are semantically more meaningful than deep learned features, but at the same time do not require a massive number of human annotators.

\subsubsection{Image recommendation}

Some interesting use-case scenarios of image recommendation can be mentioned in the fashion domain (e.g., recommending clothes) and the cultural heritage domain (e.g., recommending paintings in museums). For fashion, recommendation can be performed in two main manners: finding a piece of clothing that matches a given garment image shown to the system as a visual query (such as two pairs of jeans which are similar to each other considering their visual appearance) and finding the clothing, which complements the given query (such as recommending a pair of jeans that match a

\footnotetext{
5 http://www.pandora.com/about/mgp.

6 Pandora might also use automatically extracted content (and other) features in their system, but the MGP is arguable the approach for which Pandora is best known.
} 
shirt). The authors in McAuley et al. (2015) propose a CB-MMRS which provides personalized fashion recommendations by considering the visual appearance of clothes. The main novelty, besides focusing on this novel fashion recommendation scenario, is examining the visual appearance of the items under investigation to overcome the CS problem.

The authors of Bartolini et al. (2013) propose a multimedia (image_videodocument) recommender platform to address the cultural heritage domain: in particular, a recommender system to provide personalized visiting paths to tourists visiting the Paestum ruins, one of the major Greco-Roman cities in the South of Italy. The proposed system is able to uniformly combine heterogeneous multimedia data and to provide context-aware recommendation techniques. This paper provides interesting insights for building context-aware multimedia systems using content information, with explicit focus on contextualization. The authors exploit high-level metadata extracted in an automatic or semi-automatic manner from low-level (signal-level) features and compare it with user preferences. The main shortcoming of this research is the lack of an experimental study on a larger multimedia dataset.

Visual descriptors have also been used in restaurant recommendation systems by the authors of Chu and Tsai (2017), in which images collected from a restaurant-based social platform were first processed by an SVM-based image classification system that used both low-level and deep features and split the images into four classes, indoor, outdoor, food and drink images, based on the idea that these different categories of pictures may have different influences on restaurant recommendation. This content-based approach was used to successfully enhance the performance of matrix factorization, Bayesian personalized ranking matrix factorization and FM approaches.

In our approach, we follow a strategy that also recognizes the importance of low-level content (visual and audio) for movie recommendation and leverages it for new item CS movie recommendation.

\subsubsection{Video recommendation}

As one of the earliest approaches to the problem of video recommendations, the authors of Yang et al. (2007) and Mei et al. (2007, 2011) propose a video recommender system named "Video Reach". Given an online video and related information (query, title, tags and surrounding text), the system recommends relevant videos in terms of multimodal relevance and user feedback. Two types of user feedback are leveraged: browsing behavior and playback on different portions of the video (the latter is specific to Mei et al. 2011). These approaches are interesting from the perspective of using multimodal video content (audio, visual, and textual) and a fusion scheme based on user behavior. However, they have some limitations as well. Firstly, according to the properties required by the attention fusion function, the proposed Video Reach system filters out videos with low textual similarity to ensure that all videos are more or less relevant and then only calculates the visual similarity of the filtered videos; this may result in losing important information. Secondly, it uses only one type of visual feature, namely the basic color histogram. Thirdly, an empirical set of weights is chosen to serve as importance weights in a linear feature/modality fusion; for example, the 
textual keywords are given a much higher weight than the visual and aural keywords, without investigating the opposite arrangement. Although the authors show that this assumption is sufficient to make recommendation via adjusting weights, it is not clear what effect such an empirical assumption has.

In our approach, we introduce a video recommendation system that leverages all video properties (i.e., audio, visual, and textual) and an effective fusion method based on canonical correlation analysis (CCA) to exploit the complementary information between modalities in order to produce more powerful combined descriptors. More importantly, we propose an approach for new item recommendation that leverages the collaborative knowledge about warm items for the CBF of cold items, using the combined descriptors.

\subsection{Feature weighting for cold-start recommender systems}

Relying on CBF algorithms to address cold items has two main drawbacks: firstly, it is limited by the availability and quality of item features, and secondly, it is difficult to connect the content and collaborative information. One way to build a hybrid of content and collaborative information is via feature weighting. We focus on feature weighting rather than on other types of hybrids because we aim to build a hybridization strategy that can be easily applied to a CBF model. Feature weighting algorithms can be either embedded methods, which learn feature weights as part of the model training, or wrapper methods, which learn weights in a second phase on top of an already available model. Examples of embedded methods are user-specific feature-based similarity models (UFSM) (Elbadrawy and Karypis 2015) and factorized bilinear similarity models (FBSM) (Sharma et al. 2015). Among embedded methods, the main drawbacks are the complex training phase and a sensitivity to noise due to the strong coupling of features and interactions. UFSM learns a personalized linear combination of similarity functions, known as global similarity functions for cold-start top-N item recommendations. UFSM can be considered a special case of FM (Elbadrawy and Karypis 2015; Rendle 2012). FBSM was proposed as an evolution of UFSM that aims to discover relations among item features instead of building user-specific item similarities. The model builds an item-item similarity matrix which models how how well a feature of an item interacts with all the features of the second item.

Wrapper methods, meanwhile, rely instead on a two-step approach by learning feature weights on top of an already available model. An example of this is leastsquare feature weights (LFW) (Cella et al. 2017), which learns feature weights from a SLIM item-item similarity matrix using a simpler model than FBSM:

$$
\operatorname{sim}(i, j)=\mathbf{f}_{i}^{T} \mathbf{D} \mathbf{f}_{j}
$$

where $f$ is the feature vector of an item and $D$ is a diagonal matrix having as dimension the number of features. Another example of a wrapper method is HP3 (Bernardis et al. 2018), which builds a hybrid recommender on top of a graph-based collaborative model. A generalization of LFW has recently been published by the authors in Ferrari Dacrema et al. (2018). They demonstrate the effectiveness of wrapper methods in 
learning from a wider variety of collaborative models and present a comparative study of some state-of-the-art algorithms. Their paper further shows that wrapper methods with no latent factor component (i.e., matrix V, as in FBSM) tend to outperform others. In our approach, we therefore choose to adopt this simpler model, as it combines good recommendation quality with fast training time.

Similar strategies are available for matrix factorization models. Collective matrix factorization (Singh and Gordon 2008) allows the joint factorization of both collaborative and content data, which is applied in Saveski and Mantrach (2014) to propose local collective embedding, a joint matrix factorization that enforces the manifold structure exhibited by the collective embedding in the content data as well as allowing collaborative interactions to be mapped to topics. An example of a wrapper method is attribute to feature mapping (Gantner et al. 2010), an attribute-aware matrix factorization model which maps item features to its latent factors via a two-step approach. All previous approaches rely on the availability of some descriptors for each item, which in some cases can be an issue.

Other proposals to address the CS problem make use of other relations between users or items, i.e., social networks. For example, the authors in Zhang et al. (2010) use social tags to enrich the descriptions of items in a user-tag-object tripartite graph model; while the authors in Ma et al. (2011) instead use a social trust network to enrich the user profile. Another example is Victor et al. (2008), where authors analyze the impact of the connections on the quality of recommendations. While this group of techniques shows promising results, it is still limited by the fact that obtaining finegrained and accurate features is a complex and time-consuming task. Moreover, those other existing relationships might not always be available or meaningful for the target domain. See Elahi et al. (2018) for a good and general introduction to recommendation complicating scenarios (e.g., the CS problem).

In this work, we adopt feature weighting techniques because they have shown promising results in recent years to the point of becoming the current state of the art.

\subsection{Contributions of this work}

The work at hand builds on foundations and results realized in our previous work, but considerably extends it. We therefore present in the following our novel contributions, and connect them to previous work.

In Deldjoo et al. (2015a, b, 2016a,d), Elahi et al. (2017) and Cremonesi et al. (2018), we proposed a CB-MRS that implements a movie filter according to average shot length (measure of camera motion), color variation, lighting key (measure of contrast), and motion (measure of object and camera motion). The proposed features were originally used in the field of multimedia retrieval for movie genre classification (Rasheed et al. 2005) and have a stylistic nature which is believed to be in accordance with applied media aesthetics (Zettl 2013) for conveying communication effects and simulating different feelings in the viewers. For this reason, these features were named mise-en-scène features.

Since full movies can be unavailable, costly or difficult to obtain, in Deldjoo et al. (2016d) it was studied whether movie trailers can be used to extract mise-en-scène 
visual features. The results indicated that they are indeed correlated with the corresponding features extracted from full-length movies and that feeding the features extracted from movie trailers and full movies into a similar CB-MRS results in a comparable quality of recommendations (both superior to the genre baseline). The main shortcoming of this work is that it used a small dataset for evaluation (containing only 167 movies and the corresponding trailers). Additionally, the number of visual features was limited (only five features, cf. Rasheed et al. 2005). Due to these restrictions, the generalizability of our findings in Deldjoo et al. (2016d) may be limited; also see Sect. 6.3 for a discussion of limitations.

In Deldjoo et al. (2016b, c, 2018d) we specifically addressed the under-researched problem of combining visual features extracted from movies with available semantic information embedded in metadata or collaborative data available in users' interaction patterns in order to improve offline recommendation quality. To this end, for multimodal fusion (i.e., fusing features from different modalities) in Deldjoo et al. (2016b), for the first time, we investigated adoption of an effective data fusion technique named canonical correlation analysis (CCA) to fuse visual and textual features extracted from movie trailers. A detailed discussion about CCA can be found in Sect. 3.2. Although a small number of visual features were used to represent the trailer content (similar to Deldjoo et al. 2016d), the results of offline recommendation using $14 \mathrm{~K}$ trailers suggested the merits of the proposed fusion approach for the recommendation task. In Deldjoo et al. (2018d) we extended (Deldjoo et al. 2016b) and used both low-level visual features (color- and texture-based) using the MPEG-7 standard together with deep learning features in a hybrid CF and CBF approach. The aggregated and fused features were ultimately used as input for a collective sparse linear method (SLIM) (Ning and Karypis 2011) method, generating an enhancement for the CF method. While the results for each of these two features improved the genre and tag baselines, the best results were achieved with the CCA fusion approach. Although (Deldjoo et al. 2018d) significantly extended the previous works (Deldjoo et al. 2016b) both in terms of the content and the core recommendation model, it ignored the role of the audio modality in the entire item modeling.

Finally, in Deldjoo et al. (2016c), we used factorization machines (FM) (Rendle 2012) as the core recommendation technique. FM is a general predictor working with any real valued feature vector and has the power of capturing all interactions between variables using factorized parameters. FM was used specifically with the goal of encoding the interactions between mise-en-scène visual features and metadata features for the recommendation task. Please note that in the present work, we neither use FM nor SILM, specifically because one of the main contributions of the work at hand is to propose and simulate a novel technique for new item recommendation for which FM or SLIM are not applicable.

In a different research line, in Elahi et al. (2017), we designed an online movie recommender system which incorporates mise-en-scène visual features for the evaluation of recommendations by real users. We performed an offline performance assessment by implementing a pure CB-MRS with three different versions of the same algorithm, respectively based on (i) conventional movie attributes, (ii) mise-en-scène visual features, and (iii) a hybrid method that interleaves recommendations based on the previously noted features. As a second contribution, we designed an empirical study 
and collected data regarding the quality perceived by the users. Results from both studies showed that the introduction of mise-en-scène, together with traditional movie attributes, improves the quality of both offline and online recommendations. However, the main limitation of Elahi et al. (2017) is that we used basic late fusion by interleaving the recommendations to combine recommendations generated by different CBF systems.

In summary, although we achieved relevant progress, some limitations of our previous work remain unsolved: (i) solely visual and/or text modalities were considered, forgetting the rich audio information (e.g., conversations or music); (ii) better fusion techniques are required to fully exploit the complementary information from (several) modalities; (iii) visual content can be represented with richer descriptors; and (iv) the recommendation model used was either a CBF model based on KNN or a $\mathrm{CBF}+\mathrm{CF}$ model based on SLIM, both of which are not capable to deal with new item CS scenarios.

In this paper, we enhance these previous achievements and go beyond the state of the art in the following directions:

1. We propose a multimodal movie recommendation system which exploits established multimedia aesthetic-visual features; block-level audio features; state-ofthe-art deep visual features; and $i$-vectors audio features. Apart from the use of automated content descriptors, the system uses as input movie trailers instead of complete movies, which makes it more versatile, as trailers are more readily available than full movies. We show that the proposed CB-MRS outperforms the traditional use of metadata. To the best of our knowledge, this has not previously been achieved, existing systems being limited to the use of either visual and/or textual modalities (Deldjoo et al. 2016c, d, 2017a) or basic low-level descriptors (Yang et al. 2007; Mei et al. 2011);

2. We propose a practical solution to the CS new-item problem where user behavior data are unavailable, and therefore neither CF nor CBF using usergenerated content are applicable. Our solution consists of a two-step approach named collaborative-filtering-enriched content-based filtering ( $\mathrm{CFeCBF}$ ) to leverage the collaborative knowledge about warm items and exploit it for CBF on cold items.

3. To achieve multimodal MRS, we adopt an early fusion approach using canonical correlation analysis (CCA), which was successfully tested in our previous works (Deldjoo et al. 2016b, 2018d) for combining heterogeneous features extraced from different modalities (audio, visual and textual). CCA is often used when two types of data (feature vectors in training) are assumed to correlate. We hypothesize that this is relevant in the movie domain and that combining audio, visual, and textual data enriches the recommendations.

4. We evaluate the quality of the proposed movie genome descriptors by two comprehensive wide and articulated empirical studies: (i) a system-centric experiment to measure the offline quality of recommendations in terms of accuracy-related metrics, i.e., mean average precision (MAP) and normalized discounted cumulative gain (NDCG); and beyond-accuracy metrics (Kaminskas and Bridge 2016), i.e., list diversity, distributional diversity, and coverage; (ii) a user-centric online 


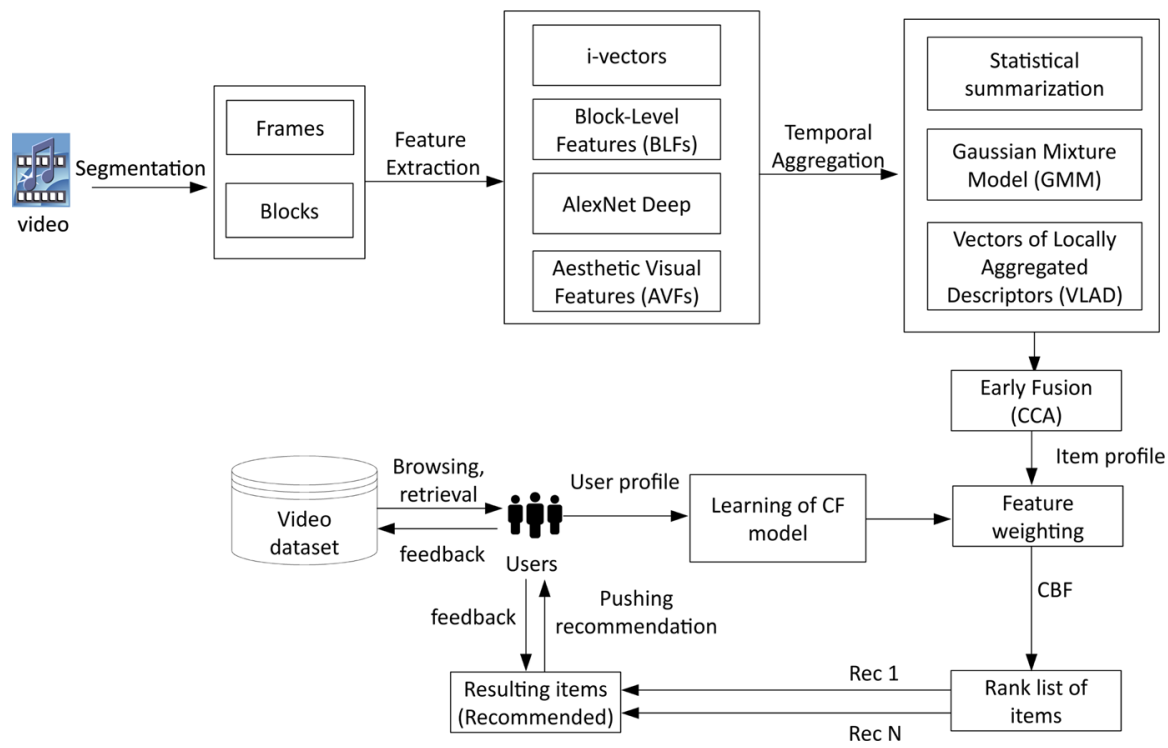

Fig. 1 The proposed collaborative-filtering-enriched content-based filtering $(\mathrm{CFeCBF})$ movie recommender system framework

experiment involving 101 users, computing different subjective metrics, including relevance, satisfaction, and diversity.

5. We publicly release the resources of this work to allow researchers to test their own recommendation models. The dataset was already released partly in Deldjoo et al. (2018c) while the code is now available on Github. ${ }^{7}$

\section{Proposed recommendation framework}

The main processing stages involved in our proposed CFeCBF-MRS are presented in Fig. 1. As previously mentioned, the only input information, apart from the collaborative one, is the movie trailers. First, we perform pre-processing that consists of decomposing the visual and audio channels into smaller and semantically more meaningful units. We use frame-level and block-level segmentation for the audio channel. For video, we use the frames captured at $1 \mathrm{fps}$. The next step consists of computing meaningful content descriptors (cf. Sect. 3.1), namely: (i) multimediaaudio and visual features; and (ii) metadata-movie genres. Features are aggregated temporally using different video-level aggregation techniques, such as statistical summarization, Gaussian mixture models (GMM), and vectors of locally aggregated descriptors (VLAD) (Jégou et al. 2010). Features are fused by using the early fusion method CCA (cf. Sect. 3.2). At this stage, each video is represented by a feature vector of fixed length, which is referred to as the item profile. A collaborative recommender is trained on all available user-item interactions in order to model the correlations

\footnotetext{
7 https://github.com/MaurizioFD/CFeCBF.
} 
encoded in users' interaction patterns, using the similarity of ratings as an indicator of similar preference. As the last step, the $\mathrm{CFeCBF}$ weighting scheme is trained on the given item profile and collaborative model to discover the hybrid feature weights. The learned feature weights are then applied to a $\mathrm{CBF}$ recommender able to provide recommendations for cold items. Each of these steps is detailed in the following sections.

\subsection{Rich item descriptions to model the movie genome}

Similar to biological DNA, which represents a living being, multimedia content information can be seen as the genome of video recommendation, i.e., the footprint of both content and style. In this section, we present the rich content descriptors integrated into the proposed movie recommendation system to boost its performance. These features were selected based on their effectiveness in representing multimedia content in various domains and comprise both audio and visual features (Deldjoo et al. 2018b, c).

\subsubsection{Audio features}

The exploited audio features are inspired by the fields of speech processing and music information retrieval (MIR) and by their successful application in MIR-related tasks, including music retrieval, music classification, and music recommendation (Knees and Schedl 2016). We investigate two kinds of audio features: (i) block-level features (Seyerlehner et al. 2011) which consider chunks of the audio signal known as blocks and are therefore capable of exploiting temporal aspects of the signal; and (ii) $i$-vector features (Eghbal-Zadeh et al. 2015) which are extracted at the level of audio segments using audio frames. Both approaches eventually model the feature at the level of the entire audio piece; by aggregating the individual feature vectors across time.

Block-level features We extract block-level features (BLF) from larger audio segments (several seconds long) as proposed in Seyerlehner et al. (2010). They can capture temporal aspects of an audio recording and have been shown to perform very well in audio and music retrieval and similarity tasks (Seyerlehner et al. 2011) and can be considered state of the art in this domain.

The BLF framework (Seyerlehner et al. 2010) defines six features. These capture spectral aspects (spectral pattern, delta spectral pattern, variance delta spectral pattern), harmonic aspects (correlation pattern), rhythmic aspects (logarithmic fluctuation pattern), and tonal aspects (spectral contrast pattern). The feature extraction process in the block-level framework is illustrated in Fig. 2. Based on the spectrogram, blocks of fixed length are extracted and processed one at a time. The block width defines how many temporally ordered feature vectors comprise a block. The hop size is used to account for possible information loss due to windowing. After having computed the feature vectors for each block, a global representation is created by aggregating the feature values along each dimension of the individual feature vectors via a summarization function, which is usually expressed as a percentile, as illustrated in Fig. 3. A more technical and algorithmic discussion can be found in Seyerlehner et al. (2010). The extraction process results in a 9948-dimensional feature vector per video. 


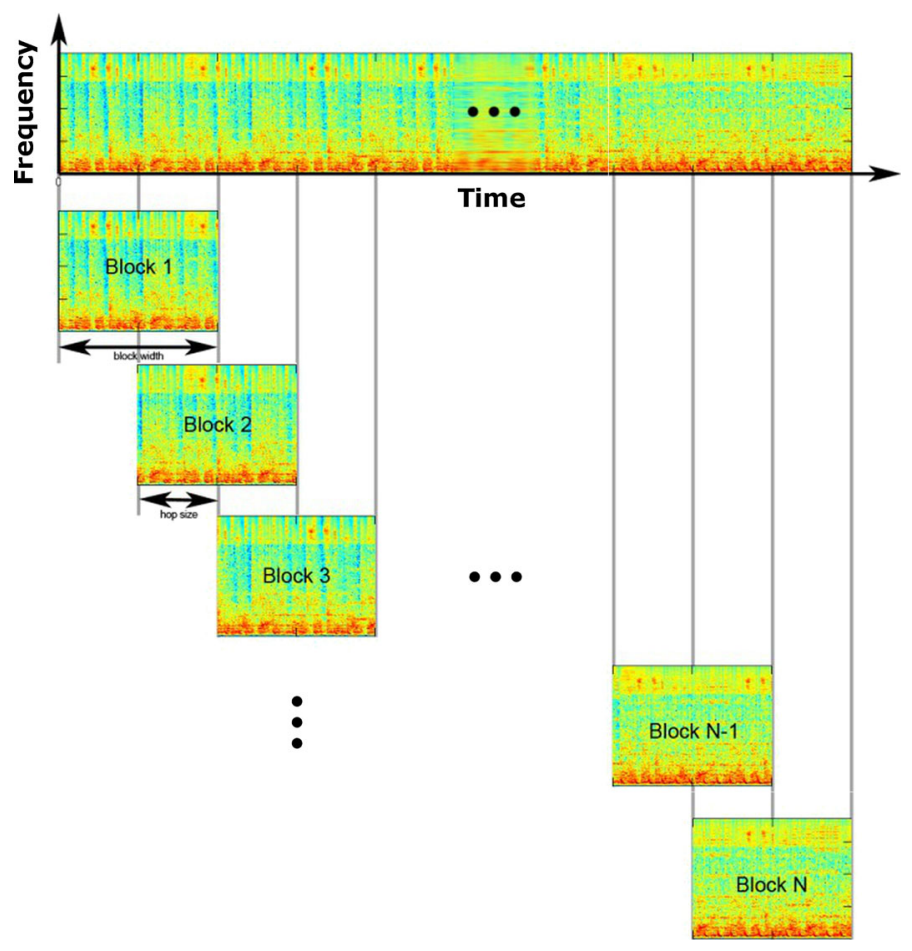

Fig. 2 Overview of the feature extraction process in the block-level features (BLF), according to Seyerlehner et al. (2010)

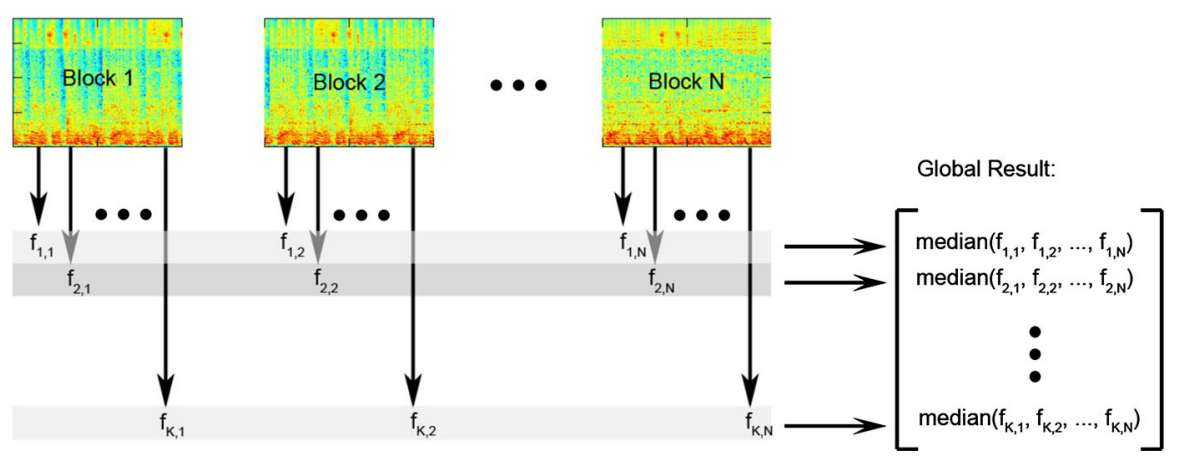

Fig. 3 Obtaining a global feature representation from individual blocks in the block-level framework, according to Seyerlehner et al. (2010)

I-vector features I-vector is a fixed-length and low-dimensional representation containing rich acoustic information, which is usually extracted from short segments (typically from $10 \mathrm{~s}$ to $5 \mathrm{~min}$ ) of acoustic signals such as speech, music, and acoustic scene. The i-vector features are computed using frame-level features such as melfrequency cepstral coefficients (MFCCs). In a movie recommendation system, we define total variability as the deviation of a video clip representation from the average 

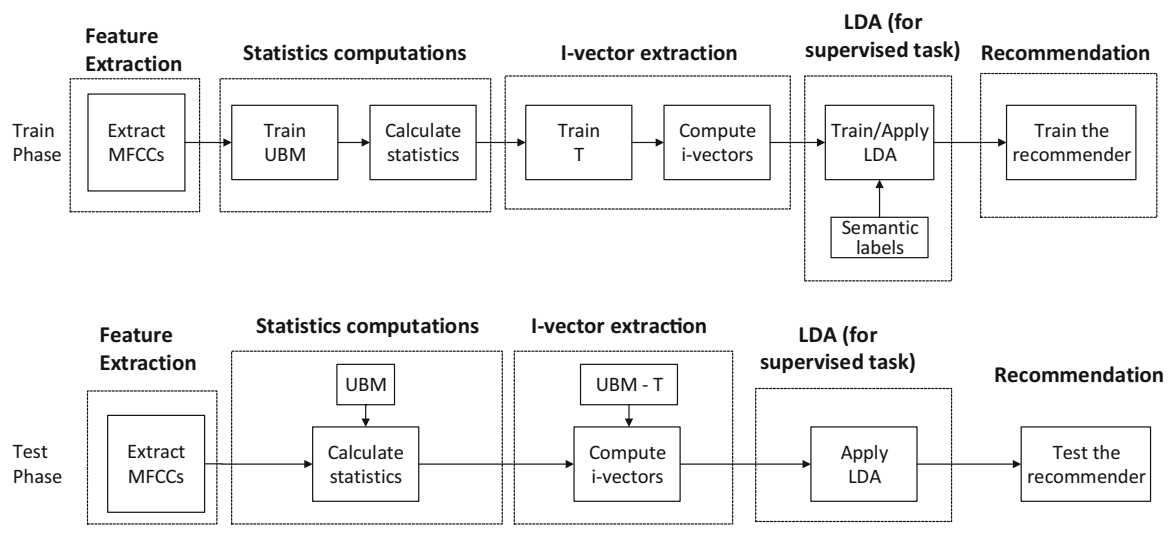

Fig. 4 Block diagram of i-vector FA pipeline for, both, supervised and unsupervised approaches

representation of all video clips. I-vectors are latent variables that capture total variability to represent how much an audio excerpt is shifted from the average clip. The main idea is to first learn a universal background model (UBM) to capture the average distribution of all the clips in the acoustic feature space using a dataset containing a sufficient amount of data consisting of different movie clips. The UBM is usually a Gaussian Mixture Model (GMM) and serves as a reference to measure the amount of shift for each segment where the i-vector is the estimated shift.

The block-diagram of the i-vector pipeline, from frame-level feature extraction to i-vector extraction and finally to recommendation, is shown in Fig. 4. The framework can be decomposed into several stages: (i) Frame-level feature extraction MFCCs have proven to be useful features for many audio and music processing tasks (Logan et al. 2000b; Ellis 2007; Eghbal-Zadeh et al. 2015). They provide a compact representation of the spectral envelope are also a musically meaningful representation (Eghbal-Zadeh et al. 2015), and are used to capture acoustic scenes (Eghbal-Zadeh et al. 2016). Even though it is possible to use other features (Suh et al. 2011), we avoid the challenges involved in feature engineering and instead focus on the timbral modeling technique. We used a 20-dimensional MFCCs feature; (ii) Computation of Baum-Welch statistics In this step, we collect sufficient statistics by adapting UBM to a specific segment. This is a process in which a sequence of MFCC feature is represented by the Baum-Welch (BW) statistics (0-th and 1-st order Baum-Welch statistics) (Lei et al. 2014; Kenny 2012) using a GMM as prior; (iii) I-vector extraction I-vector extraction refers to the extraction of total factors from BW statistics. This step reduces the dimensionality of the movie clip representations and improves the representation for a recommendation task; (iv) Recommendation Recommendation is effected by integrating the extracted i-vector features in a CBRS.

During the training phase, the UBM is trained on the items in the training dataset and is used as an external knowledge source for the test dataset. In the testing step, test i-vectors are extracted using the models from the training step and the MFCCs of the test set. In the supervised approach, these i-vectors are projected by LDA in the training step. For the i-vector extraction, we used 20-dimensional MFCCs. For the items in the training set (in each fold), we trained a UBM with either 256 or 512 
Gaussian components and a different dimensionality of latent factors (40, 100, 200, 400). We performed a hyper-parameter search and reported the best results obtained over fivefold cross-validation for each evaluation metric.

\subsubsection{Visual features}

The visual features we selected for our experiments were previously used in other domains, including image aesthetics, media interestingness, object recognition, and affect classification. We selected two types of visual features: (i) aesthetic visual features, a set of features mostly associated with media aesthetics, and (ii) deep learning features extracted from the fc7 layer of the AlexNet deep neural network, initially developed for visual object recognition, but extended and used in numerous other domains. Several aggregation methods were also performed with these features, with the goal of obtaining video-level descriptors from the frame-level set of extracted features.

Aesthetic-visual features the three groups of features and their early fusion combinations were aggregated in a standard statistical aggregation scheme based on mean, median, variance, and median absolute deviation. In a work discussing the measurement of coral reef aesthetics, the authors in Haas et al. (2015) propose a set of features inspired by the aesthetic analysis of artwork ( $\mathrm{Li}$ and Chen 2009) and photographic aesthetics (Datta et al. 2006; Ke et al. 2006). This collection of features is derived from related domains, such as photographic style, composition, and the human perception of images, and was grouped into three general features types: color-related, texture-related and object-related.

The color-related features have 8 main components. The first elements consist of the average channel values extracted from the HSL and HSV color spaces. A colorfulness measure was created by calculating the Earth Mover's Distance, Quadratic Distance and standard deviation between two distributions: the color frequency in each of the 64 divisions of the RGB spectrum and an equal reference distribution. The hue descriptors contained statistical calculations for pixel hues: number of hues present, number of significant hues for the image etc. The hue models are based on the distance between the current picture and a set of nine hue models considered appealing for humans inspired by the models presented in Matsuda (1995). The brightness descriptor calculates statistics regarding image brightness, including average brightness values and brightness/contrast across the image. Finally, average HSV and HSL values were calculated while taking into account the main focus region and rule of thirds compositional guideline (Obrador et al. 2010).

The texture-related features have 6 components. The edge component calculates statistics based on edge distribution and energy, while the texture component calculates statistics based on texture range and deviation. Also entropy measures were calculated on each channel of the RGB color space, generating a measure of randomness. A three-level Daubechies wavelet transform (Daubechies 1992) was calculated for each channel of the HSV space along with the values for the average wavelet. A final texture component was based on the low depth-of-field photographic composition rule, according to the method described by Datta et al. (2006). 
The object-related features have 11 components. These components are mostly based on the largest segments in an image obtained through the method proposed in Datta et al. (2006), which is based on the k-means clustering algorithm. The area, centroids, values for the hue, saturation, and value channels, average brightness values, horizontal and vertical coordinates, mass variance and skewness for the largest, and therefore most salient, segments each constitutes a component of this feature type. Color spread and complementarity also represented a component, while the last component calculates hue, saturation, and brightness contrast between the resulting segments.

As previously mentioned, this set of features is highly correlated to the human observer, some components being heavily based on psychological or aesthetic aspects of visual communication. For example, the hue model component calculates the distance between the hue model of a certain image and models considered appealing to humans, inspired by the work of Matsuda (1995). Also, some general rules of photographic style were used, rules previously shown to have a high impact on human aesthetic perception, therefore generating more pleasant images and videos (Krages 2012). For example, the authors in Liu et al. (2010) modify images in order to achieve a better aesthetic score, one of the rules applied for this optimization being the rule of thirds.

We used these features in our experiments, both separated into the three main feature types (color, texture and object) and in an early fusion concatenated descriptor for each image in the video. Regarding the aggregation method, we used four standard statistical aggregation schemes based on mean, median, variance and median absolute deviation.

Deep-learning features Deep neural networks have become an important part of the computer vision community, gathering interest and gaining importance as their results started performing better than more traditional approaches in different domains. The ImageNet Large Scale Visual Recognition Competition (ILSVRC) gives the opportunity to test different object recognition algorithms on the same dataset, consisting of a subset of 1.2 million images and 1000 different classes taken from the ImageNet ${ }^{8}$ database. The AlexNet (Krizhevsky et al. 2010) deep neural network was the winner of the competition in 2012, achieving a top-5 error rate of $15.3 \%$ - a significant improvement over the second-best entry - that year. The authors also ran experiments on the ILSVRC 2010 dataset, concluding that the top- 1 and top-5 error rates of $37.5 \%$ and $17 \%$ were again improvements on previous state-of-the-art approaches. One of the novelties introduced by this network was the ReLU (Rectified Linear Units) nonlinearity output function, which was able to achieve faster training times than networks working with more standard functions like $f(x)=\tanh (x)$ or $f(x)=\left(1+e^{-x}\right)^{-1}$, instead using $f(x)=\max (0, x)$.

AlexNet consists of 5 convolutional layers and 3 fully connected layers, ending with a final, 1000-dimensional softmax layer. The input of the network consists of a $224 \times 224 \times 3$ image, therefore requiring the original image to be resized if the resolution is different. The five convolutional layers have the following structure: the first layer has 96 kernels of size $11 \times 11 \times 3$; the second, 256 kernels of size $5 \times 5 \times 48$;

\footnotetext{
8 http://www.image-net.org/.
} 
the third, 384 kernels of size $3 \times 3 \times 256$; the fourth, 384 kernels of size $3 \times 3 \times 192$; and the final, fifth convolutional layer, 256 kernels of size $3 \times 3 \times 192$. The fully connected layers all have 4096 neurons, and the output of the final one is fed into a softmax layer that creates a distribution for the 1000 labeled classes. This generates a network with 60 million parameters and 650, 000 neurons; thus, in order to reduce overfitting on the original dataset, some data augmentation solutions were employed, including image translations, horizontal reflections, and the alteration of the intensity of the RGB channels and a dropout technique (Hinton et al. 2012).

Given the good performance of the fc7 layer in tasks related to human preference, we chose to extract the outputs of this layer for each frame of our videos, thus obtaining a 4096-dimensional descriptor for each image. We then obtain a video-level descriptor through two types of aggregation methods: standard statistical aggregation, where we calculate the mean, median, variance, and median absolute deviation, and VLAD (Jégou et al. 2010) aggregation followed by PCA for dimensional reduction, with three different sizes for the visual word codebook: $k \in\{32,64,128\}$.

\subsubsection{Metadata features}

We also use two types of editorial metadata features to serve as baselines: movie genre and cast/crew features.

Genre features For every movie, genre features are used to serve as metadata baselines. Genre Features (18 categories): Action, Adventure, Animation, Children's, Comedy, Crime, Documentary, Drama, Fantasy, Film Noir, Horror, Musical, Mystery, Romance, Sci-Fi, Thriller, War, and Western. The final genre feature vector is a binary, 18-dimensional vector. ${ }^{9}$

Cast/crew features For every movie, the corresponding cast and crew have been downloaded from TMDB ${ }^{10}$ using the available API and movie ID mapping provided by Movielens20M. The feature vector contains $162 \mathrm{~K}$ Boolean features. Each movie is associated, on average, with 25 features.

\subsection{Multimodal fusion}

Two main paradigms of fusion exist in the literature of multimedia processing (Snoek et al. 2005): (i) late fusion which generates separate candidate results created by different systems and fuses them into a final set of results; the main limitation of late fusion methods is that they do not consider the correlation among features and are computationally more expensive during training; (ii) early fusion which tries to map multiple feature spaces to a unified space, in which conventional similarity-based evaluation can be conducted.

Motivated by the above, in current work we exploit a multimodal early fusion method based on canonical correlation analysis (CCA) that was successfully tested in

\footnotetext{
9 While presenting the results, we will use the genre metadata as the baseline for evaluation, as it is prevalent in the domain. Furthermore, we refrain from using user-generated metadata such as tag features in this work, since in a new item CS situation these features cannot exist.

10 https://www.themoviedb.org/.
} 
our previous works (Deldjoo et al. 2016b, 2018d). CCA is a technique for joint fusion and dimensionality reduction across two or more (heterogeneous) feature spaces, which is often used when two set of data are believed to have some underlying correlation. We hypothesize that this is relevant in movie domain and combining audio, visual and textual data enriches the recommendations and training. Additionally, since the focus of the recommendation model in our work is on a CF-enriched CBF model (see Sect. 3.3), we have realized that currently the proposed method functions better with a lower size of the feature vectors. As CCA reduces the dimensionality of the final descriptor, it is is leveraged greatly in the proposed recommendation framework. Finally, CCA can be pre-computed and used in an off-the-shelf manner making it a convenient descriptor in offline experiments (as opposed to late fusion methods Deldjoo et al. 2018b).

We review the concept of CCA here for our methodology. Let $X \in \mathbb{R}^{p \times n}$ and $Y \in \mathbb{R}^{q \times n}$ be two sets of features in which $p$ and $q$ are the dimensions of features extracted from the $n$ items. Let $S_{x x}=\operatorname{cov}(x) \in \mathbb{R}^{p \times p}$ and $S_{y y}=\operatorname{cov}(y) \in \mathbb{R}^{q \times q}$ be the within-set and $S_{x y}=\operatorname{cov}(x, y) \in R^{p \times q}$ be the between-set covariance matrix. Let us further define $S \in \mathbb{R}^{(p+q) \times(p+q)}$ as the overall covariance matrix-a complete matrix which contains information about associations between pairs of featuresrepresented as follows:

$$
S=\left(\begin{array}{ll}
S_{x x} & S_{x y} \\
S_{y x} & S_{y y}
\end{array}\right)=\left(\begin{array}{ll}
\operatorname{cov}(x) & \operatorname{cov}(x, y) \\
\operatorname{cov}(y, x) & \operatorname{cov}(y)
\end{array}\right)
$$

The aim of CCA is to identify a pair of linear transformations, represented by $X^{*}=W_{x}^{T} X$ and $Y^{*}=W_{y}^{T} Y$, that maximizes the pairwise correlation across two feature sets given by

$$
\arg \max _{W_{x}, W_{y}} \operatorname{corr}\left(X^{*}, Y^{*}\right)=\frac{\operatorname{cov}\left(X^{*}, Y^{*}\right)}{\operatorname{var}\left(X^{*}\right) \cdot \operatorname{var}\left(Y^{*}\right)}
$$

where $\operatorname{cov}\left(X^{*}, Y^{*}\right)=W_{x}^{T} S_{x y} W_{y}$ and $\operatorname{var}\left(X^{*}\right)=W_{x}^{T} S_{x x} W_{x}$ and $\operatorname{var}\left(Y^{*}\right)=$ $W_{y}^{T} S_{y y} W_{y}$.

In order to solve the above optimization problem, we use the maximization procedure described in Haghighat et al. (2016). The CCA model parameters $W_{x}$ and $W_{x}$ are learned on trained items (warm items) and leveraged both in the training and test phases. We investigate two ways to perform fusion: (i) via concatenation (abbreviated by 'ccat') and (ii) via summation (abbreviated by 'sum') of the transformed features.

\subsection{The cold-start recommendation model}

The core recommendation model in our system is a standard pure CBF system using Eq. (4) to compute similarities between different pair of videos:

$$
\operatorname{sim}(i, j)=\frac{\mathbf{f}_{i} D \mathbf{f}_{j}}{\left\|\mathbf{f}_{i}\right\|_{F}^{2}\left\|\mathbf{f}_{j}\right\|_{F}^{2}}
$$


where $\mathbf{f}_{i} \in \mathbb{R}^{n_{F}}$ is the feature vector for video $i,\|\|_{F}^{2}$ is the Frobenius norm and $n_{F}$ is the number of features. We are interested in finding the diagonal weight matrix $D \in \mathbb{R}^{n_{F} \times n_{F}}$, which represents the importance of each feature.

An underlying assumption is that a CF model will achieve much higher recommendation quality than $\mathrm{CBF}$ and will be better able to capture the user's point-of-view. We use a $C F$ model to learn $D$, cast into the following optimization problem:

$$
\underset{\mathbf{D}}{\operatorname{argmin}}\left\|\mathbf{S}^{(\mathbf{C F})}-\mathbf{S}^{(\mathbf{D})}\right\|_{F}^{2}+\alpha\|\mathbf{D}\|_{F}^{2}+\beta\|\mathbf{D}\|
$$

where $\mathbf{S}^{(\mathbf{C F})}$ is the item-item collaborative similarity matrix from which we want to learn, $\mathbf{S}^{(\mathbf{D})}$ is the item-item hybrid similarity metric presented via Eq. (4), D is the feature weight matrix, $\alpha$ and $\beta$ are the weights of the regularization terms.

We call this model collaborative-filtering-enriched content-based filtering (CFeCBF). The optimal $\mathbf{D}$ is learned via machine learning, applying stochastic gradient descent with Adam (Kingma and $\mathrm{Ba}$ 2014), which is well suited for sparse and noisy gradients. The code is available on Github. ${ }^{11} \mathrm{CFeCBF}$ is a wrapper method for feature weighting; therefore, it does not learn weights while building the model but rather relies on a previously trained model and then learns feature weights as a subsequent step. Since the model we rely on is collaborative, we can only learn weights associated with features that occur in warm items. This affects how well the algorithm can perform in scenarios where the available features are too sparse; in this case, the number of features appearing in s but not in warm items will tend to increase, reducing the number of parameters in the model.

It is important to point out that while it will be possible to learn a zero collaborative similarity for items having a common feature, it will not be possible to learn anything for items with no common features. Therefore, content-based similarity poses a hard constraint on the extent to which collaborative information can be learned. As contentbased similarity is a function of the item features, the sparser this matrix is the less information will be learnable from a collaborative model. This could be a challenge when using Boolean features that tend to be sparse, but much less of one when using real-valued attributes like the multimedia descriptors, which result in dense feature vectors. A consequence of this is that the success of applying CFeCBF on a given dataset depends not only on how accurate the collaborative model is, but also on whether its similarity structure, resulting from the items having common features, is sufficiently compatible with that of the content-based model. ${ }^{12} \mathrm{CFeCBF}$ requires a two-step training procedure. In the first step, we aim to find the optimal hyper-parameters for a collaborative model by training it on warm items and selecting the optimal hyperparameters via cross-validation. Since we want a single hyper-parameters set, not one for each fold, we chose those with the best average recommendation quality across all the training folds.

\footnotetext{
11 https://github.com/MaurizioFD/CFeCBF.

12 As our experiments have showed, the best collaborative similarity will not necessarily yield the best weights.
} 
Fig. 5 User interactions' item-wise split; A contains warm items while B contains s and refers to a subset of the users
Items

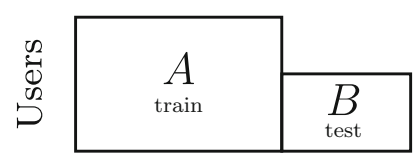

Once the collaborative model is available, the second step is to learn weights by solving the minimization problem described in Eq. (5). As the purpose of this method is to learn $\mathbf{D}$, or feature weights, the optimal hyper-parameters for the machine learning phase are chosen via a cold item split to improve the CBF on new items. Figure 5 shows how a cold item split is performed: split $A$ represents the warm items, that is, items for which we have interactions and that we can use to train the collaborative model, and split $B$ represents cold items that we use only for testing the weights. All reported results for pure $\mathrm{CBF}$ and $\mathrm{CFeCBF}$ are reported on split $B$.

\section{Experimental study A: Offline experiment}

In this experiment, we investigate offline recommendation in cold- and warm-start scenarios. The specific experimental setup is presented in the following section.

\subsection{Data}

We evaluated the performance of the proposed MRS on the MovieLens-20M (ML20M) dataset (Harper and Konstan 2016), which contains user-item interactions between users and an up-and-running movie recommender system. We employ fivefold cross-validation (CV) in our experiments by partitioning the items in our dataset into 5 non-overlapping subsets (item-wise splitting of the user-rating matrix). Different folds will have different cold items. Similar to Adomavicius and Zhang (2012), we built the test split by randomly selecting 3000 users, each having a minimum of 50 ratings in their rating profile, in order to speed up the experiments on the many feature sets. The items those users interacted with will be considered cold items; see split B in Fig. 5. The remaining items and interactions will be part of the training set. The reported results are referred to split B. Meanwhile, split A is used to perform parameter tuning. The characteristics of the data split are shown in Table 2. The significantly higher number of ratings per item in the training set (A) is due to the fact that it contains more users, and hence more interactions, than the test set (B).

Table 2 Characteristics of the evaluation dataset used in the offline study: $|\mathcal{U}|$ is the number of users, $|\mathcal{I}|$ the number of items, $|\mathcal{R}|$ the number of ratings

\begin{tabular}{lllllll}
\hline ML-20M & $|\mathcal{U}|$ & $|\mathcal{I}|$ & $|\mathcal{R}|$ & $\frac{|\mathcal{R}|}{|\mathcal{U}|}$ & $\frac{|\mathcal{R}|}{|\mathcal{I}|}$ & $\frac{|\mathcal{R}|}{|\mathcal{I}| \cdot|\mathcal{U}|}$ (density) \\
\hline Train (A) & $138 \mathrm{~K}$ & $12.6 \mathrm{~K}$ & $10 \mathrm{M}$ & 72.46 & 793.65 & 0.0057 \\
Test (B) & $3 \mathrm{~K}$ & $4.8 \mathrm{~K}$ & $212 \mathrm{~K}$ & 70.67 & 44.16 & 0.0144 \\
\hline
\end{tabular}




\subsection{Objective evaluation metrics}

For assessing performance in the offline experiments, we compute the two categories of metrics, accuracy metrics (cf. Sect. 4.2.1) and beyond-accuracy metrics (cf. Sect. 4.2.2). The name and definition of the specific metrics computed is provided in the corresponding sections.

\subsubsection{Accuracy metrics}

Mean average precision (MAP) is a metric that computes the overall precision of a recommender system, based on precision at different recall levels (Li et al. 2010). It is computed as the arithmetic mean of the average precision (AP) over the entire set of users in the test set, where AP is defined as follows:

$$
A P=\frac{1}{\min (M, N)} \sum_{k=1}^{N} P @ k \cdot \operatorname{rel}(k)
$$

where $\operatorname{rel}(k)$ is an indicator signaling if the $k$ th recommended item is relevant, i.e., $\operatorname{rel}(k)=1$, or not, i.e., $\operatorname{rel}(k)=0 ; M$ is the number of relevant items; and $N$ is the number of recommended items in the top $N$ recommendation list. Note that AP implicitly incorporates recall, because it considers relevant items not in the recommendation list. Finally, given the AP equation, MAP will be defined as follows:

$$
M A P=\frac{1}{|U|} \sum_{u \in|U|} A P_{u}
$$

Normalized discounted cumulative gain (NDCG) is a measure for the ranking quality of the recommendations. This metric was originally proposed to evaluate the effectiveness of information retrieval systems (Järvelin and Kekäläinen 2002). It is nowadays also frequently used for evaluating music recommender systems (Liu and Yang 2008; Park and Chu 2009; Weimer et al. 2008). Assuming that the recommendations for user $u$ are sorted according to the predicted rating values in descending order, $D C G_{u}$ is defined as follows:

$$
D C G_{u}=\sum_{i=1}^{N} \frac{r_{u, i}}{\log _{2}(i+1)}
$$

where $r_{u, i}$ is the true rating (as found in test set $T$ ) for the item ranked at position $i$ for user $u$, and $N$ is the length of the recommendation list. Since the rating distribution depends on users' behavior, the DCG values for different users are not directly comparable. Therefore, the cumulative gain for each user should be normalized. This is done by computing the ideal DCG for user $u$, denoted as $I D C G_{u}$, which is the $D C G_{u}$ value that provides the best possible ranking, obtained by ordering the items by true ratings in descending order. Normalized discounted cumulative gain for user $u$ is then computed as follows: 


$$
N D C G_{u}=\frac{D C G_{u}}{I D C G_{u}}
$$

Finally, the overall normalized discounted cumulative gain $N D C G$ is computed by averaging $N D C G_{u}$ over the entire set of users.

\subsubsection{Beyond-accuracy metrics}

The purpose of a recommender system is not only to recommend relevant items to the user based on their past behavior but also to facilitate exploration of the catalogue, helping to discover new items that the user might find interesting. Beyond-accuracy metrics try to assess if the recommender is able to diversify its recommendations for different users and leverage the whole catalogue or if it is focused on just a few highly popular items. In this study, we focus on the following measures:

Coverage of a recommender system is defined as the proportion of items which have been recommended to at least one user (Herlocker et al. 2004):

$$
\text { coverage }=\frac{|\hat{I}|}{|I|}
$$

where $|I|$ is the cardinality of the test item set and $|\hat{I}|$ is the number of items in $I$ which have been recommended at least once. Recommender systems with lower coverage are limited in the number of items they recommend.

Intra-list diversity Is another important beyond-accuracy measure. It gauges the extent to which recommended items are different from each other, where difference can relate to various aspects, e.g., genre, style or composition. Diversity can be defined in several ways. One of the most common is to compute the pairwise distance between all items in the recommendation set, either averaged (Ziegler et al. 2005) or summed (Smyth and McClave 2001). In the former case, the diversity of a recommendation list $L$ is calculated as follows:

$$
\operatorname{Intra} L(L)=\frac{\sum_{i \in L} \sum_{j \in L \backslash i} d_{i s t_{i, j}}}{|L| \cdot(|L|-1)}
$$

where $d i s t_{i, j}$ is some distance function defined between items $i$ and $j$. Common choices are inverse cosine similarity (Ribeiro et al. 2012), inverse Pearson correlation (Vargas and Castells 2011), or Hamming distance (Kelly and Bridge 2006). In our experiments we report a diversity computed using the genre of the movies and cosine similarity.

Inter-list diversity or inter-user diversity measures the uniqueness of different users recommendation lists (Zhou et al. 2010). Given two users $i$ and $j$, and their recommendation list $L$, the inter-list distance can be calculated by:

$$
\operatorname{Inter} L\left(L_{i}, L_{j}\right)=1-\frac{q\left(L_{i}, L_{j}\right)}{|L|}
$$


where $q\left(L_{i}, L_{j}\right)$ is the number of common items in recommendation lists of length $|L|$. $\operatorname{Inter} L\left(L_{i}, L_{j}\right)=0$ indicates identical lists and $\operatorname{Inter} L\left(L_{i}, L_{j}\right)=1$, completely different ones. The mean distance is obtained by averaging $\operatorname{Inter} L\left(L_{i}, L_{j}\right)$ over all pairs of users such that $i \neq j$.

A model which tends to frequently recommend the same set of items will result in similar recommendation lists and low diversity, whereas a recommender better able to tailor its recommendations to each user will exhibit higher diversity (Zhou et al. 2010). In this respect, inter-list diversity and intra-list diversity are complementary. Consider a Top Popular recommender (i.e., one that recommends the most popular items). Its recommendations might have high intra-list diversity if they involve movies with different characteristics; therefore, a user will perceive them as diverse. However, all users will receive the same recommendations and both item coverage and inter-list diversity will be very low.

While an increase in diversity can indicate that the recommender is better able to offer personalized recommendations, it should be taken into account that the lowest diversity, and item coverage, will be obtained by always recommending the same items, whereas the highest will be obtained by a random recommender. This is another example of the accuracy-diversity trade-off.

In order to better understand how much the proposed techniques truly contribute towards more diverse and idiosyncratic recommendations across all users, in addition to the above beyond-accuracy metric, we also computed the metrics entropy, Gini coefficient, and Herfindahl (HHI) index (Adomavicius and Kwon 2012). These metrics provide different means for measuring distributional dispersion of recommended items across all users, and are therefore referred to as aggregate diversity. If recommendations are concentrated on a few popular items, the recommender will have low coverage and low diversity in terms of entropy and HHI but high Gini Index. If recommendations are more equally spread out across all candidate items, the recommender will exhibit high diversity and coverage but low Gini Index (Adomavicius and Kwon 2012). These metrics provide an overview of the recommender system from a systemwide point of view and are useful for assessing its behavior when deployed on a real, business-oriented system.

The distributional dispersion metrics are defined as follows:

$$
\begin{aligned}
& \text { Entropy }=-\sum_{i \in I} \frac{\operatorname{rec}(i)}{r e c_{t}} \cdot \ln \frac{\operatorname{rec}(i)}{r e c_{t}} \\
& \text { Gini }- \text { index }=\sum_{i=1}^{|I|} \frac{2 i-|I|-1}{|I|} \cdot \frac{\operatorname{rec}(i)}{r e c_{t}} \\
& \text { Herfindahl }- \text { index }=1-\frac{1}{r e c_{t}^{2}} \sum_{i \in I} r e c(i)^{2}
\end{aligned}
$$

where $\operatorname{rec}(i)$ refers to the number of times item $i$ has been recommended over all users, $r e c_{t}$ the total number of recommendations (i.e., cutoff value times the number of test users), $I$ the cold items set, and $|I|$ its cardinality. Note that while the Gini 
index and Herfindahl index have a value range between 0 and 1, Shannon entropy is not bounded by 1 .

\subsection{Collaborative filtering model}

Following the results of Ferrari Dacrema et al. (2018) we chose as collaborative model RP3beta (Paudel et al. 2017) which demonstrated a very competitive recommendation quality at a very small computational cost, since it does not require ML. RP3beta is a graph-based algorithm which models a random walk between two sets of nodes, users and items. Each user is connected to the items he/she interacted with and each item is similarly connected to the users. The model consists of an item-item similarity matrix which represents the transition probability between the two items, computed directly via the graph adjacency matrix, easily obtainable from the URM. The similarity values are are elevated at a coefficient alpha and divided by each item's popularity elevated to a coefficient beta, the latter acting as a reranking phase which takes the popularity bias into account.

\subsection{Hyper-parameter tuning}

The proposed approach requires two types of parameter tuning. Firstly, it is necessary to train and tune the CF model. Since we want a single optimal hyper-parameter set we train the $\mathrm{CF}$ recommender on all the train folds separately and then select the hyperparameters corresponding to the best average recommendation quality on all folds, measured with MAP. This constitutes a robust validation and testing methodology, and reduces the risk to overfit. Each fold will be associated with its own collaborative model since different folds will correspond to different cold items split. Secondly, the tuning of the hyper-parameters of the feature weighting machine learning is performed in a similar way, again optimizing MAP. We searched the optimal hyper-parameters via a Bayesian search (Antenucci et al. 2018) using the implementation of Scickitoptimize. ${ }^{13}$ As for different aggregation methods designed for the audio and visual features, we chose the best performing ones with regards to the metric under study.

\subsection{Overall computational time and complexity}

In this section, we provide general information regarding runtimes and overall computational complexity of the subsystems in the proposed framework.

Regarding the extraction of the visual features, this process performs above the real-time frame rate of the movies ( 25 or 30 frames per second). We have performed feature extraction on a computer with Intel Xeon E5-1680 processor with 8 cores, 16 threads and a base frequency of $3.00 \mathrm{GHz}, 192 \mathrm{~GB}$ RAM and an NVIDIA 1080TI GPU card with 3584 CUDA cores. While the extraction of AlexNet features was handled by the GPU, with an average speed of 62.8 processed frames per second, the extraction

13 https://scikit-optimize.github.io/. 
of the aesthetic visual features was done on the CPU, in parallel, using 7 of the 8 available cores and recording an average speed of 38.3 processed frames per second.

The feature weighting phase has a low computational complexity as it requires, for each epoch, to compute the gradient for each collaborative similarity value and compute the prediction error by using the item features. It is therefore linear in terms of both the number of descriptors and in terms of the number of similarities which in turn grows quadratically on the items. In terms of runtime, on an Intel Xeon E3-1246 $3.50 \mathrm{GHz}$ with $32 \mathrm{~GB}$ RAM, learning the weights on the descriptors of length 200 takes $15 \mathrm{~min}$ on a single core, including the time required to perform the validations needed by early stopping.

\subsection{Performance analysis: accuracy metrics}

The experiments performed in Study A can be divided into four different categories, as presented in Table 3: baseline experiments using the genre and cast/crew metadata features, both editorially created (cf. Sect. 3.1.3) ${ }^{14}$; unimodal experiments using traditional and state-of-the-art (SoA) audio and visual features (cf. Sect. 3.1); content-based multimodal experiments, where the proposed canonical correlation analysis (CCA) is used as an early fusion method (cf. Sect. 3.2); and finally, collaborative-filtering enhanced multimodal experiments, where the systems from the previous multimodal experiments are enhanced through the use of collaborative filtering (cf. Sect. 3.3). In the latter two, multimodal, categories, we report and analyze the performance of all combinations from the proposed unimodal features and the genre baseline. ${ }^{15}$

As a general observation, we see that the unimodal visual and audio features constantly outperform the baseline metadata systems. The best performance is obtained by Deep visual features, improving the genre baseline by $53.0 \%$ in terms of NDCG and by $42.8 \%$ in terms of MAP. Even the lowest performing unimodal feature, i.e., ivector, still achieves a $14.4 \%$ increase for NDCG and a $7.1 \%$ increase for MAP over the baseline. We further observe that the Deep feature outperforms the traditional AVF feature in the visual category, while in the audio category, the reverse pattern occurs, i.e., the traditional BLF feature has a better performance than the i-vector audio feature for both metrics.

As presented in Sect. 3.2, our multimodal approaches use CCA as a fusion method. We compared the CCA approach with a simple concatenation method, as well as with a weighted late fusion Borda count method, as described in Deldjoo et al. (2018b). We chose CCA as our early fusion method because all results were better for the CCA approach. For example, in the case of the i-vec + genre multimodal combination, CCA achieved a 9.5\% MAP increase and a 20.2\% NDCG increase over the simple concatenation method in the pure $\mathrm{CBF}$ approach, while in the $\mathrm{CFeCBF}$ approach,

\footnotetext{
14 Note that we could not use tags as a feature in Study A, since the tags available in this dataset are user-generated. For cold items, no interactions with users have occurred yet, so no tags could be provided. While it could be possible the users added tags without providing a rating, this does not solve the underlying problem as it presumes a kind of interaction. Therefore the available tags for each items will be related to its popularity, some items will acquire tags easily while others may have none for quite some time.

15 Note that we use the genre features as the main baseline due to their widespread usage and the fact that genre and cast had similar performance in almost all reported metrics.
} 
Table 3 Performance of various features: i-vector (Audio), BLF (Audio), Deep (Visual), and AVF (Visual), editorial-metadata, in terms of accuracy metrics NDCG and MAP. For fusion, we report the results for the CCA fusion variation (either ccat or sum) that lead to the best performance (cf. Sect. 3.2). The features (or feature combination) which outperform genre significantly are shown in bold $(p<0.05)$

\begin{tabular}{|c|c|c|c|}
\hline \multirow[t]{2}{*}{ Feature name } & \multirow[t]{2}{*}{ CCA fusion } & \multicolumn{2}{|c|}{ Accuracy Metrics } \\
\hline & & NDCG & MAP \\
\hline \multicolumn{4}{|l|}{ Base } \\
\hline $\operatorname{cast}(\mathrm{EM})$ & - & 0.0088 & 0.0041 \\
\hline genre(EM) & - & 0.0083 & 0.0042 \\
\hline \multicolumn{4}{|l|}{ Unimodal } \\
\hline i-vec (A: SoA) & - & 0.0095 & 0.0045 \\
\hline BLF (A: traditional) & - & 0.0111 & 0.0055 \\
\hline Deep (V: SoA) & - & 0.0127 & 0.0060 \\
\hline AVF (A: traditional) & - & 0.0108 & 0.0047 \\
\hline \multicolumn{4}{|l|}{ Multimodal (pure CBF) } \\
\hline i-vec + Genre $(\mathrm{A}+\mathrm{EM})$ & sum & 0.0101 & 0.0046 \\
\hline i-vec + Deep $(A+V)$ & sum & 0.0089 & 0.0042 \\
\hline $\mathrm{i}-\mathrm{vec}+\mathrm{AVF}(\mathrm{A}+\mathrm{V})$ & ccat & 0.0077 & 0.0038 \\
\hline $\mathrm{i}-\mathrm{vec}+\mathrm{BLF}(\mathrm{A})$ & sum & 0.0094 & 0.0046 \\
\hline AVF + Genre $(\mathrm{V}+\mathrm{EM})$ & sum & 0.0086 & 0.0039 \\
\hline $\mathrm{AVF}+\mathrm{BLF}(\mathrm{V}+\mathrm{A})$ & sum & 0.0091 & 0.0052 \\
\hline $\mathrm{AVF}+\operatorname{Deep}(\mathrm{V})$ & sum & 0.0086 & 0.0041 \\
\hline Deep + Genre $(V+$ EM $)$ & sum & 0.0078 & 0.0038 \\
\hline Deep + BLF $(V+A)$ & sum & 0.0102 & 0.0053 \\
\hline BLF + Genre $(\mathrm{A}+\mathrm{EM})$ & ccat & 0.0090 & 0.0046 \\
\hline \multicolumn{4}{|l|}{ Multimodal (CFeCBF) } \\
\hline i-vec+Genre (A + EM) & sum & 0.0186 & 0.0078 \\
\hline i-vec + Deep $(A+V)$ & sum & 0.0176 & 0.0083 \\
\hline $\mathrm{i}-\mathrm{vec}+\mathrm{AVF}(\mathrm{A}+\mathrm{V})$ & sum & 0.0121 & 0.0062 \\
\hline $\mathrm{i}-\mathrm{vec}+\mathrm{BLF}(\mathrm{A})$ & ccat & 0.0177 & 0.0083 \\
\hline AVF+Genre (V + EM) & sum & 0.0192 & 0.0097 \\
\hline $\mathrm{AVF}+\mathrm{BLF}(\mathrm{V}+\mathrm{A})$ & sum & 0.0102 & 0.0048 \\
\hline $\mathbf{A V F}+\operatorname{Deep}(\mathbf{A}+\mathbf{V})$ & sum & 0.0191 & 0.0097 \\
\hline Deep + Genre $(\mathrm{V}+\mathrm{EM})$ & sum & 0.0117 & 0.0059 \\
\hline Deep + BLF $(A)$ & sum & 0.0111 & 0.0052 \\
\hline $\mathrm{BLF}+\mathrm{Genre}(\mathrm{A}+\mathrm{EM})$ & ccat & 0.0120 & 0.0059 \\
\hline
\end{tabular}

$A$ audio, $V$ visual, $E M$ editorial metadata

the CCA fusion method achieved a $151.6 \%$ increase in terms of MAP and a $181.8 \%$ increase in terms of NDCG. These results confirm not only that CCA fusion produces good results on its own but also that it increases the power of collaborative filtering approaches by heavily reducing the size of the feature vector. Furthermore, the use of an early fusion method such as CCA allows us to easily create systems that outperform the late fusion method mentioned in Deldjoo et al. (2018b), in both accuracy metrics. 
For the multimodal CBF approach, we observe that the CCA fusion of the best performing unimodal audio and visual features (i.e., Deep and BLF) leads to the best multimodal results. More precisely, Deep + BLF achieves a $22.8 \%$ improvement over the baseline (0.0102 vs. 0.0083 ) in terms of NDCG and a $26.1 \%$ increase in terms of MAP (0.0053 vs. 0.0042). Similarly, the combination i-vec + genre performed strongly, improving on the baseline by $21.6 \%$ for NDCG (0.0101 vs. 0.0083$)$ and $9.5 \%$ for MAP (0.0046 vs. 0.0042$)$. This result was surprising, since both individual features, genre and i-vec, had a weaker performance in the unimodal experiment. In fact, in all genre combinations, such as AVF + genre, BLF + genre, and i-vec + genre, we can see an improvement in performance. This suggests that the genre feature has an information-complementary nature with other modalities, which can be leveraged using the CCA fusion. However, the combination of Deep + genre is an exception, as one can observe a decrease in performance. This may be due to the correlation between the two.

The multimodal CFeCBF approach aims to enable the recommendation of cold items by leveraging collaborative knowledge of warm items. The proposed method was applied on CCA multimodal approaches, as presented in the CBF multimedia approach. Looking at the performance globally, one can observe that the CFeCBF multimodal approach improves the pure CBF multimodal systems in all 10 combinations along NDCG and in 8 combinations along MAP; the few non-improved feature combinations, i.e., AVF + BLF and Deep + BLF, already performed well in pure CBF experiments. For NDCG, the average growth factor is $67 \%$, with the minimum equal to $7 \%$ for Deep + BLF and the maximum equal to $123 \%$ for AVF + Genre. For MAP, the average growth factor is $68 \%$, with the minimum equal to $-7 \%$ for AVF + BLF and the maximum equal to $148 \%$ for $\mathrm{AVF}+$ Genre. When compared with the genre baseline, the proposed CFeCBF method improves the features, on average, by $79.75 \%$ for MAP and $72.6 \%$ for NDCG.

One final step was taken for the validation of these results, namely performing the significance tests as pairwise comparisons between the best performing systems and the best performing baseline genre. For both NDCG and MAP metrics, we performed statistical significance tests using the multiple comparison test provided by the statistical and machine learning toolbox in MATLAB ${ }^{16}$ (function multcompare()), in which we adopted Fisher's least significant difference to compensate for multiple tests when performing all pairwise comparisons. Detailed information about the test can be found in Sheskin (2003). The three best performing systems, $i$-vec + genre, $A V F+$ genre, and $A V F+$ Deep, show significant improvements over the baseline with $p<0.05$, where the improvement along NDCG is $124.1 \%, 131.33 \%$, and $130.12 \%$, respectively, and that along MAP equal to $85.7 \%, 130.12 \%$, and $130.12 \%$, respectively. These results indicate the effectiveness of the proposed approach in dealing with very different kinds of features and its ability to embed collaborative knowledge in a $\mathrm{CBF}$ recommender. In particular, the systems showing significant improvements have lower dimensionality for the descriptors than the others. This suggests that learning feature weights becomes harder as the number of dimensions increases. Applying

\footnotetext{
$\overline{16}$ https://www.mathworks.com/help/stats/multiple-comparisons.html.
} 
dimensionality reduction techniques is therefore beneficial when dealing with very long descriptors.

\subsection{Performance analysis: beyond-accuracy metrics}

In this section, we report the results for beyond-accuracy metrics. The results are summarized in Table 4 (reports diversity metrics computed on the various recommendation lists: inter-list diversity and intra-list diversity) and Table 5 (reports all the aggregate diversity metrics, which are instead computed on the overall number of times each item was recommended to any user: Item coverage, Shannon entropy, Gini index, and Herfindahl Index).

From Table 4, we can observe that intra-list diversity (intraL) exhibits similar values across all cases. As previously mentioned, this diversity is computed with respect to the genre of movies, so a higher diversity would mean recommendations of heterogeneous genres, while a lower diversity would mean recommendations of the same genre. Following this definition, we expect that a recommender based only on genre as a feature will exhibit the lowest intraL diversity, which is in fact what we do observe. If we consider that as baseline value, we can see that all other features-metadata, unimodal or multimodal - achieve slightly higher diversity while not penalizing recommendation accuracy; this increase is significant in all cases. In terms of inter-list diversity (interL), results are more varied. We can see that multimodal recommenders, both pure $\mathrm{CBF}$ and hybrid $\mathrm{CFeCBF}$, yield higher diversity in most cases, meaning that given any two users, the average number of items they have in common in their recommendation lists is going to be lower. The increased InterL diversity for CFeCBF is statistically significant in almost all cases. This suggests that multimodal recommenders will be less prone to concentrate their recommendations on a small subset of items.

From Table 5, we can see the results for aggregate diversity metrics. Note that while greater diversity will result in higher values for Item coverage, Shannon entropy, and Herfindahl Index, it will drive Gini index closer to zero. These metrics allow us to look at the recommender from the point of view of the whole system instead of that of the user, which is important when deploying recommenders as a part of a business model. We first focus on Item coverage, which tells us the portion of cold items the system was able to recommend. We can immediately see that the baseline recommenders using metadata have poor coverage: only half of the available items were recommended at least once. Most models based on multimodal features, instead, exhibit significantly higher coverage-up to more than $90 \%$, meaning they are able to explore the catalogue much better without sacrificing recommendation quality. The other metrics measure the number of times each item has been recommended. Compared to the coverage, they provide the additional information about the number of occurrences. Within a certain coverage value, the distribution of items can be very different. For example, in the case of a Top Popular recommender in a warm item scenario, the final coverage will be higher than the length of the recommendation list because some users will already have already interacted with those items and therefore other, less popular, items will be recommended to them. Distribution diversity metrics allow us to determine the extent 
Table 4 Performance of various features in terms of beyond-accuracy metrics for list diversity. For fusion, we report the results for the CCA fusion variation (either ccat or sum) that lead to the the best performance (cf. Sect. 3.2). Results in bold show the features (or feature combinations) that outperform genre significantly $(p<0.05)$ along the respective metric

\begin{tabular}{|c|c|c|c|}
\hline \multirow[t]{2}{*}{ Feature name } & \multirow[t]{2}{*}{ CCA fusion } & \multicolumn{2}{|c|}{ List diversity } \\
\hline & & IntraL & InterL \\
\hline \multicolumn{4}{|l|}{ Base } \\
\hline cast (EM) & - & 0.8990 & 0.8794 \\
\hline genre (EM) & - & 0.8886 & 0.9035 \\
\hline \multicolumn{4}{|l|}{ Unimodal } \\
\hline i-vec (A: SoA) & - & 0.8994 & 0.9322 \\
\hline BLF (A: traditional) & - & 0.8994 & 0.9522 \\
\hline Deep (V: SoA) & - & 0.8992 & 0.8641 \\
\hline AVF (A: traditional) & - & 0.8994 & 0.9528 \\
\hline \multicolumn{4}{|l|}{ Multimodal (pure CBF) } \\
\hline i-vec + Genre $(\mathrm{A}+\mathrm{EM})$ & sum & 0.8965 & $\mathbf{0 . 9 5 7 7}$ \\
\hline i-vec + Deep $(A+V)$ & sum & 0.8994 & 0.9602 \\
\hline $\mathrm{i}-\mathrm{vec}+\mathrm{AVF}(\mathrm{A}+\mathrm{V})$ & ccat & 0.8994 & 0.7682 \\
\hline $\mathrm{i}-\mathrm{vec}+\mathrm{BLF}(\mathrm{A})$ & ccat & 0.8995 & 0.8772 \\
\hline $\mathrm{AVF}+$ Genre $(\mathrm{V}+\mathrm{EM})$ & (sum, ccat) & 0.8927 & 0.9536 \\
\hline $\mathrm{AVF}+\mathrm{BLF}(\mathrm{V}+\mathrm{A})$ & (sum, ccat) & 0.8995 & 0.8724 \\
\hline $\mathrm{AVF}+\operatorname{Deep}(\mathrm{V})$ & ccat & 0.8994 & 0.6890 \\
\hline Deep + Genre $(V+$ EM) & ccat & 0.8964 & 0.9633 \\
\hline Deep + BLF $(V+A)$ & ccat & 0.8995 & 0.9548 \\
\hline $\mathrm{BLF}+\mathrm{Genre}(\mathrm{A}+\mathrm{EM})$ & ccat & 0.8969 & 0.9616 \\
\hline \multicolumn{4}{|l|}{ Multimodal $(\mathrm{CFeCBF})$} \\
\hline i-vec + Genre $(\mathrm{A}+\mathrm{EM})$ & sum & 0.8981 & 0.9304 \\
\hline i-vec + Deep $(A+V)$ & sum & 0.8995 & 0.9535 \\
\hline $\mathrm{i}-\mathrm{vec}+\operatorname{AVF}(\mathrm{A}+\mathrm{V})$ & (ccat, ssum) & 0.8995 & 0.9584 \\
\hline $\mathrm{i}-\mathrm{vec}+\mathrm{BLF}(\mathrm{A})$ & sum & 0.8995 & 0.9533 \\
\hline $\mathrm{AVF}+$ Genre $(\mathrm{V}+\mathrm{EM})$ & (sum, ccat) & 0.8992 & 0.7532 \\
\hline $\mathrm{AVF}+\mathrm{BLF}(\mathrm{V}+\mathrm{A})$ & sum & 0.8995 & $\mathbf{0 . 9 3 7 3}$ \\
\hline $\mathrm{AVF}+\operatorname{Deep}(\mathrm{V})$ & ccat & 0.8995 & 0.9549 \\
\hline Deep + Genre $(\mathrm{V}+\mathrm{EM})$ & (ccat, sum) & 0.8983 & 0.9361 \\
\hline Deep + BLF $(\mathrm{V}+\mathrm{A})$ & ccat & 0.8995 & 0.9599 \\
\hline $\mathrm{BLF}+\mathrm{Genre}(\mathrm{A}+\mathrm{EM})$ & ccat & 0.8986 & 0.9396 \\
\hline
\end{tabular}

$A$ audio, $V$ visual, $E M$ editorial metadata

to which the recommender is trying to diversify its recommendations. As an example, consider the 4 cases having coverage between 94.5 and $96.5 \%$, with an interval of just $2 \%$ of all items. These cases exhibit a Gini index varying between .65 and .78 , meaning that there is a difference in the number of times those items were recommended. In particular, the increase in coverage was accompanied in this case by more unbalanced, and therefore less diverse, item occurrence. 
Table 5 Performance of various features in terms of beyond-accuracy metrics for aggregate diversity. Results in bold show the features (or feature combinations) that outperform genre significantly $(p<0.05)$. For each feature combination, we only report the results for the CCA method that has the best performance (either ccat or sum)

\begin{tabular}{|c|c|c|c|c|c|}
\hline \multirow[t]{2}{*}{ Feature name } & \multirow[t]{2}{*}{ CCA fusion } & \multicolumn{3}{|c|}{$\underline{\text { Distributional diversity }}$} & \multirow{2}{*}{$\begin{array}{l}\text { Item } \\
\text { Cov }\end{array}$} \\
\hline & & Gini & Entropy & HHI & \\
\hline \multicolumn{6}{|l|}{ Base } \\
\hline cast (EM) & - & 0.7652 & 7.2672 & 0.9879 & 0.5348 \\
\hline genre (EM) & - & 0.7424 & 7.4525 & 0.9903 & 0.5435 \\
\hline \multicolumn{6}{|l|}{ Unimodal } \\
\hline i-vec (A: SoA) & - & 0.7055 & 8.2934 & 0.9932 & 0.9276 \\
\hline BLF (A: traditional) & - & 0.6614 & 8.5983 & 0.9952 & 0.9412 \\
\hline Deep (V: SoA) & - & 0.7992 & 7.2659 & 0.9864 & 0.6615 \\
\hline AVF (A: traditional) & - & 0.6583 & 8.6165 & 0.9952 & 0.9336 \\
\hline \multicolumn{6}{|l|}{ Multimodal (pure CBF) } \\
\hline i-vec + Genre $(A+E M)$ & sum & 0.6510 & 8.6752 & 0.9957 & 0.9431 \\
\hline i-vec + Deep $(A+V)$ & sum & 0.6283 & 8.7951 & 0.9960 & 0.9960 \\
\hline $\mathrm{i}-\mathrm{vec}+\mathrm{AVF}(\mathrm{A}+\mathrm{V})$ & ccat & 0.7794 & 6.1634 & 0.9768 & 0.2569 \\
\hline $\mathrm{i}-\mathrm{vec}+\mathrm{BLF}(\mathrm{A})$ & ccat & 0.8022 & 7.2558 & 0.9877 & 0.6412 \\
\hline $\mathrm{AVF}+$ Genre $(\mathrm{V}+\mathrm{EM})$ & ccat & 0.6754 & 8.4935 & 0.9953 & 0.8811 \\
\hline $\mathrm{AVF}+\mathrm{BLF}(\mathrm{V}+\mathrm{A})$ & (ccat, $*$ : sum) & $0.6595 *$ & 6.7429 & 0.9872 & 0.3014 \\
\hline $\mathrm{AVF}+\operatorname{Deep}(\mathrm{V})$ & (ccat) & 0.8037 & 5.7369 & 0.9689 & 0.2147 \\
\hline Deep + Genre $(\mathrm{V}+\mathrm{EM})$ & (ccat, $*$ :sum) & 0.6361 & 8.7644 & 0.9963 & $0.9388 *$ \\
\hline Deep + BLF $(\mathrm{V}+\mathrm{A})$ & (ccat) & 0.6402 & 8.7184 & 0.9954 & 0.9655 \\
\hline BLF + Genre $(A+E M)$ & (ccat) & 0.6381 & 8.7520 & 0.9961 & 0.9459 \\
\hline \multicolumn{6}{|l|}{ Multimodal (CFeCBF) } \\
\hline i-vec + Genre $(A+E M)$ & sum & 0.7232 & 8.0490 & 0.9930 & 0.7769 \\
\hline $\mathrm{i}-\mathrm{vec}+\operatorname{Deep}(\mathrm{A}+\mathrm{V})$ & sum & 0.6719 & 8.5653 & 0.9953 & $0.9275 *$ \\
\hline $\mathrm{i}-\mathrm{vec}+\operatorname{AVF}(\mathrm{A}+\mathrm{V})$ & sum & 0.6342 & 8.6499 & 0.9958 & 0.8736 \\
\hline $\mathrm{i}-\mathrm{vec}+\mathrm{BLF}(\mathrm{A})$ & sum & 0.6667 & 8.5831 & 0.9953 & 0.9299 \\
\hline $\mathrm{AVF}+$ Genre $(\mathrm{V}+\mathrm{EM})$ & ccat & 0.7742 & 6.0510 & 0.9753 & 0.2345 \\
\hline $\mathrm{AVF}+\mathrm{BLF}(\mathrm{V}+\mathrm{A})$ & (sum, *:ccat) & 0.7150 & 8.0789 & 0.9937 & $0.7664 *$ \\
\hline $\mathrm{AVF}+\operatorname{Deep}(\mathrm{V})$ & ccat & 0.6504 & 8.5740 & 0.9955 & 0.8691 \\
\hline Deep + Genre $(\mathrm{V}+\mathrm{EM})$ & sum & 0.7170 & 8.1647 & 0.9936 & 0.8258 \\
\hline Deep + BLF $(\mathrm{V}+\mathrm{A})$ & ccat & 0.6439 & 8.7395 & 0.9960 & 0.9595 \\
\hline $\mathrm{BLF}+\operatorname{Genre}(\mathrm{A}+\mathrm{EM})$ & ccat & 0.7007 & 8.2965 & 0.9939 & 0.8619 \\
\hline
\end{tabular}

$A$ audio, $V$ visual, EM editorial metadata, Entropy Shannon entropy, HHI Herfindahl, Item Cov item coverage * is used to identify which of the ccat and sum variations produced the best result

We can see how there is a significant difference between Multimodal and Base recommenders in terms of Gini index, meaning that the multimodal recommenders, both pure and hybrid, have more balanced item distribution. The combination of very high item coverage and improved distributional diversity metrics suggest that the collaborative machine learning step does not add a popularity bias to the feature 
weights, on the contrary $\mathrm{CFeCBF}$ is less subject to it than the Base recommenders. Moreover, we see that Shannon Entropy increases, meaning that the recommender is getting less "predictable" in the recommendations it will provide. This confirms what was observed in terms of interL diversity. The Herfindahl index is known to have a small value range when applied to recommender systems, as we can see in our experiments where its value ranges from 0.96 to 0.99 . Compared to the other indices, it is less sensitive to items being recommended only a few times, due to its quadratic nature, but more sensitive to items being recommended a high number of times. Its values confirm the increased diversity achievable by Multimodal recommenders in almost all cases for pure $\mathrm{CBF}$ and in all cases for hybrid $\mathrm{CFeCBF}$.

\subsection{Cold to warm item transition}

While the core of our experimental study is aimed at cold start items, in a real case scenario we expect some interactions to become available over time as the users interact with the cold items. For practical use it is interesting to assess when it is appropriate to change the recommendation model from a content based, either pure or CFeCBF, to a collaborative model. To this end we design a brief study, aiming to assess at which interaction density an item transition from cold to warm, allowing the use of CF methods.

It is already well known that, depending on the dataset, even a few interactions may be sufficient to outperform CBF approaches (Pilászy and Tikk 2009).

\subsubsection{Experimental protocol}

To simulate a realistic cold to warm transition we add some interactions to the cold items. Those interactions are taken from the original test set of that fold. Since this study requires to create a new data split, with a denser train and a sparser test set, the results here reported are not comparable to the ones reported in the previous study.

We report two different experimental settings, one preserves the popularity distribution of the items, the other does not. The reader should notice that, being sampled in different, ways, the test set of the two experiments are different and the results are not directly comparable.

Random sampling In order to preserve the statistical distribution of the interactions and the impact of the item's popularity, the new train interactions for the cold items are randomly sampled, with no constraints applied. This will result in a mixture of popular items having a few interactions and unpopular items having none. This experiment allows to assess what happens in a realistic case in which some cold items will be popular and therefore collect interactions much faster, while others will not. This is motivated by the fact that $\mathrm{CF}$ algorithms, which $\mathrm{CFeCBF}$ is learning from, are sensitive to the popularity distribution and altering such distribution will result in biased $\mathrm{CF}$ models. The original test data is sampled so that $2 \%$ of its interactions become new train data and $98 \%$ constitute the new test data. To show the behaviour at different densities, the train data is further divided in a smaller set only containing $0.5 \%$ of the original test interactions. 
Fixed number of interactions While the previous experiment models a real case scenario more accurately, it leaves open the question of how significant is the effect of the popularity bias on the results. To this end also build a different split which contains a fixed number of train interactions for the cold items. This creates an artificial popularity distribution which will change the behaviour of the $\mathrm{CF}$ model. The number of interactions we chose is 1 and 5. This will result in a perfectly balanced train set. In this case the test data is composed by the original test data minus 4 interactions for each item.

This new train data is therefore composed by the original train data plus the interactions sampled from the test set and is used to train all algorithms: $\mathrm{CBF}, \mathrm{CF}$ and $\mathrm{CFeCBF}$. The optimal parameters remain those selected in the previous phase when no interactions were available. In a real case scenario it would be impractical to run a new tuning of the model's parameters after each few interactions are added. It is instead more realistic for this tuning phase to be executed again only once a sufficient amount of new data is available.

\subsubsection{Result discussion}

The results for the random split are reported in Table 6 for both accuracy metrics and Item Coverage. ${ }^{17}$ As it is possible to see, in terms of accuracy metrics the recommendation quality of pure $\mathrm{CBF}$ remains constant as the transition progresses. $\mathrm{CFeCBF}$, instead, changes its recommendation quality, in some cases improving over the cold item case, in others not. This is due to the evolving CF model it is learning from.

The most important thing to observe is that the pure collaborative algorithm, RP3beta, is immediately able to outperform all CBF and CFeCBF models in terms of accuracy metrics. It should be noted that Movielens, the dataset from which the interactions are taken, tends to exhibit high recommendation quality for collaborative algorithms which makes this cold to warm transition very fast. Consider that Warm $0.5 \%$ corresponds to an average of $1 \times 10^{-1}$ interactions per item and Warm $2.0 \%$ of $4 \times 10^{-1}$ interactions per item. Looking at the recommendation quality alone is however misleading. In terms of diversity it is possible to see that CF has a remarkably low item coverage. This means that the $\mathrm{CF}$ algorithm is still not able to explore the catalogue, being confined to a marginal $6 \%$ of the available items. The result can be explained by the significant popularity bias of the dataset, hence a few items account for a sizable quota of the interactions, while many others have much fewer. This behaviour means that the CF model is recommending only a few popular items, being unable to recommend the vast majority of them. CF fails completely to allow the user a broad exploration of the catalogue and offers very little personalization. Moreover, if the items are not seen by the users, it will be very difficult to collect the interactions needed for them to become warm items, the risk being to keep them in a cold state for very long. $\mathrm{CFeCBF}$, on the other hand, has a very high Item Coverage, which allows a broader exploration of the catalogue, yielding to a higher probability cold items will be rated and a more effective $\mathrm{CF}$ model could be applied at a later stage.

17 For brevity we did not report all beyond accuracy metrics. 


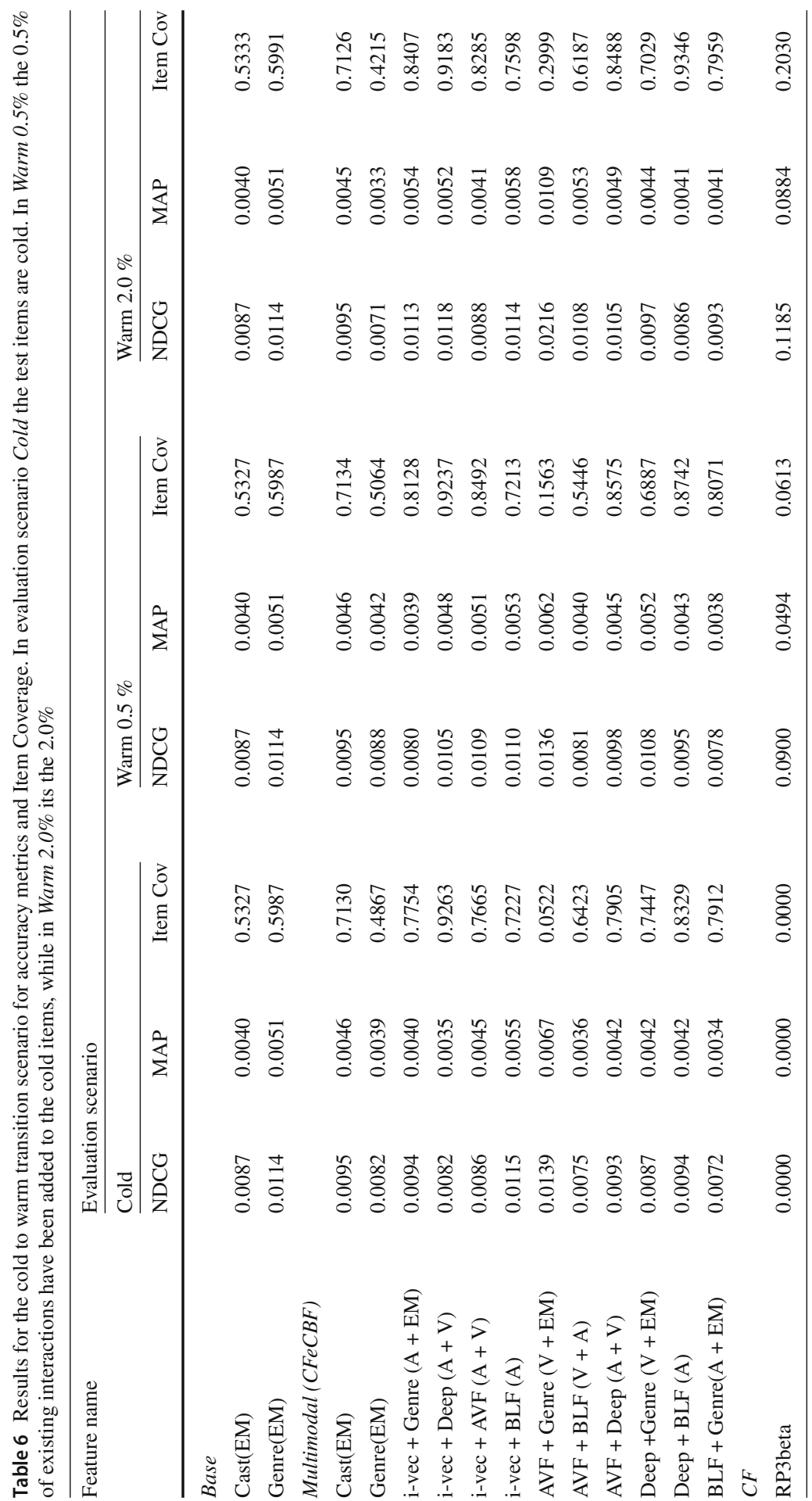


If we look at the results for the fixed number of interactions experiment in Table 7, we can observe a different behaviour. The CBF and CFeCBF models maintain their almost stable recommendation quality while $\mathrm{CF}$ increases. However, as opposed to the previous case, we can see that the $\mathrm{CF}$ advantage grows less steeply with respect to CFeCBF even though the train data is much denser, 1 and 4 as opposed to $4 \times 10^{-1}$. Moreover, the CF Item Coverage is comparable or higher than CFeCBF. This allows to state that the behaviour of the $\mathrm{CF}$ algorithm in the random sampling experiment is strongly influenced by the significant popularity bias of the dataset.

To summarize, in terms of accuracy metrics CF algorithms are able to outperform $\mathrm{CBF}$ and $\mathrm{CFeCBF}$ when even just a few interactions are available, more so if the dataset has a strong popularity bias. However $\mathrm{CBF}$ and $\mathrm{CFeCBF}$ maintain a sizable advantage in terms of diversity metrics and Item Coverage. Depending on the specific use-case or application, and therefore the desired balance between accuracy and catalogue exploration, a different strategy may be adopted. If the main focus is on accuracy, then as soon as the item has an interaction it can be considered as warm. The reader should note that, while Movielens has a high popularity bias, other datasets with a less pronounced bias will exhibit a less steep CF quality improvement. If the focus is on improving catalogue exploration to reduce the popularity bias effect then the target number of interactions per item may be pushed further.

\section{Experimental study B: insights from a preliminary user study about perceived quality}

In this section, we describe an empirical study whose goal is not to recommend new movies, as in the experimental study A, but to understand to what extent the proposed movie genome is perceived as useful when deployed in a real MRS. The developed system uses a pure $\mathrm{CBF}$ recommender based on the $\mathrm{KNN}$ algorithm and measures the utility of the recommendation as perceived by the user in terms of accuracy, novelty, diversity, level of personalization, and overall satisfaction. In this study, we intentionally avoid the discussion of hybridization and focus instead only on six unimodal recommendation approaches, classifiable in 3 categories: (i) metadata: genre and tag, (ii) audio: $i$-vectors and $B L F$, and (iii) visual: Deep features and $A V F$. We use only the unimodal recommendation schemes presented in the experimental study A. The reason for this is to avoid overloading users with too many recommendation choices, and thus to be able to obtain more reliable responses from users collectively. Note that in this study, the tags feature is considered because, as stated, the study's focus is no longer on new movie recommendation (as in study A) and tags serve as a rich semantic baseline.

Our preliminary studies in a similar direction have been published in Elahi et al. (2017) which focused on a single visual modality (Elahi et al. 2017), and in Deldjoo et al. (2018b), which used a lower number of participants (74 vs. 101). In addition, compared to Deldjoo et al. (2018b), we performed better sanity checks and removed unreliable user input. Further information is provided in the following sections. 


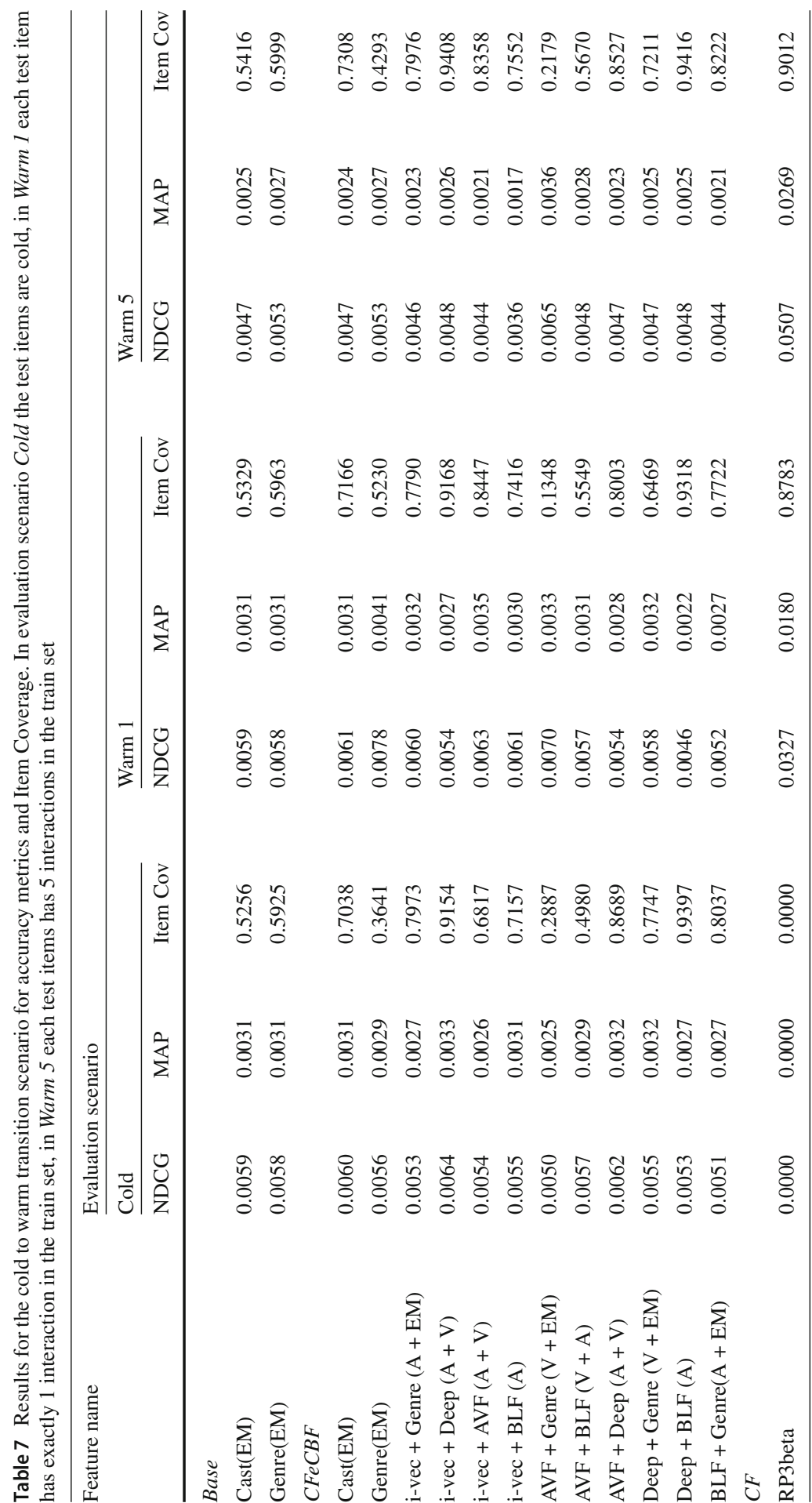




\subsection{Perceived quality metrics}

The goal of the current study is to measure how the user perceives the quality of the proposed recommender system. Perceived quality is as an indirect indicator of a recommenders potential for persuasion (Cremonesi et al. 2012). It is defined as the degree to which the users judge recommendations positively and appreciate the overall experience of the recommender system. We operationalize the notion of perceived quality in terms of five metrics (Ekstrand et al. 2014): Perceived accuracy (also called Relevance) - measures how much the recommendations match users' interests, preferences, and tastes; Satisfaction - measures global users' feelings about their experience with the recommender system; Understands me-relates to perceived personalization or the user's perception that the recommender understands their tastes and can effectively adapt to them; Novelty ${ }^{18}$-measures the extent to which users receive new (unknown) recommendations; Diversity - measures how much users perceive recommendations as different from each other, e.g., movies from different genres.

\subsection{Evaluation protocol}

To measure the user's perception of the recommendation lists according to the five quality metrics explained above, we adopt the questionnaire proposed in Knijnenburg et al. (2012). This instrument contains 22 questions to assess various aspects of the recommendation lists. For convenience, these questions are shown in Table 8. As suggested by the authors from Ekstrand et al. (2014), the questions are asked in a comparative mode instead of seeking absolute values.

We developed MISRec (Mise-en-Scène Movie Recommender), a web-based testing framework for the movie search and recommendation domain, which can easily be configured to facilitate the execution of controlled empirical studies. Some screenshots of the system are presented in Fig. 6. MISRec is powered by a pure CBF algorithm based on KNN and supports users with a wide range of functionalities common in online video-streaming services such as Netflix. ${ }^{19}$ MISRec contains the same cata$\log$ of movies used in the first study (see Sect. 4). Users can browse the catalog of movies, retrieve detailed descriptions of each, rate them, and receive recommendations. MISRec also embeds an online questionnaire system that allows researchers to easily collect quantitative and qualitative information from the user. The first prototype of MISRec was used for conducting an empirical study on the contribution of stylistic visual features to movie recommendation, and the results were published in Elahi et al. (2017). A more recent development of MISRec powered by the proposed movie genome features was published in Deldjoo et al. (2018b). An extension of the system was also developed in Deldjoo et al. (2017b) to use the system in an interactive manner e.g., for kid movie recommendation using cover photos of the movies as the system activator.

\footnotetext{
18 Note that we could not use Novelty as an evaluation criterion in study A because Novelty is defined in terms of item popularity, which is available for warm items but not for cold items.

19 https://www.netflix.com.
} 
Table 8 The list of questions (Ekstrand et al. 2014; Knijnenburg et al. 2012) used to measure the perceived quality of recommendations. Note that answers/scores given to questions marked with a + contribute positively to the final score, whereas scores to questions marked with a - are subtracted

Factor/question (W. 1. = which list, W. r. = which recommender)

\section{Percieved accuracy}

W. 1. has more movies that you find appealing? $(\mathbf{Q 1 7}+)$

W. 1. has more movies that might be among the best movies you see in the next year? $(\mathbf{Q 1 9}+)$

W. 1. has more obviously bad movie recommendations for you? (Q6 -)

W. r. does a better job of putting better movies on the left? $(\mathbf{Q 9}+)$

Diversity

W. 1. has more movies that are similar to each other? (Q22 -)

W. 1. has a more varied selection of movies? $(\mathbf{Q 7}+)$

W. 1. has movies that match a wider variety of moods? $(\mathbf{Q 1 3}+)$

W. 1. would suit a broader set of tastes? $(\mathbf{Q 2}+)$

\section{Understands me}

W. r. better understands your taste in movies? $(\mathbf{Q 1 2}+)$

W. r. would you trust more to provide you with recommendations? $(\mathbf{Q 1 8}+)$

W. r. seems more personalized to your movie taste? $(\mathbf{Q 1 4}+)$

W. r. more represents mainstream tastes instead of your own? (Q3 -)

Satisfaction

W. r. would better help you find movies to watch? $(\mathbf{Q 8}+)$

W. r. would you be more likely to recommend to your friends? $(\mathbf{Q 1 6}+)$

W. 1. of recommendations do you find more valuable? $(\mathbf{Q 1 1}+)$

W. r. would you rather have as an app on your mobile phone? $(\mathbf{Q 2 0}+)$

W. r. would better help to pick satisfactory movies? $(\mathbf{Q 1}+)$

Novelty

W. 1. has more movies you do not expect? $(\mathbf{Q 2 1}+)$

W. 1. has more movies that are familiar to you? (Q4 -)

W. 1. has more pleasantly surprising movies? (Q5 +)

W. 1. has more movies you would not have thought to consider to watch? $(\mathbf{Q 1 0}+)$

W. 1. provides most new suggestions? $(\mathbf{Q 1 5}+)$

Our main target audience is users aged between 19 and 54 who have some familiarity with the use of the web but have never used MISRec before the study (to control for the potentially confounding factor of biases or misconceptions derived from previous uses of the system). The total number of recruited subjects who also completed the task was 101 (73 male, 28 female, mean age 25.64 years, std. 6.61 years, min. 19 years, max. 54 years). Data collection were carried out mostly from master students at three universities: Politecnico Di Milano Italy, JKU Linz Austria and Politehnica di Bucharest, Romania attending the course of Recommender Systems or similarly related courses. They were trained to perform the study, were given written instructions on the evaluation procedure, and were regularly supervised by $\mathrm{Ph} . \mathrm{D}$. students and a PostDoc researcher during their activities. The interaction begins with a sign-up 

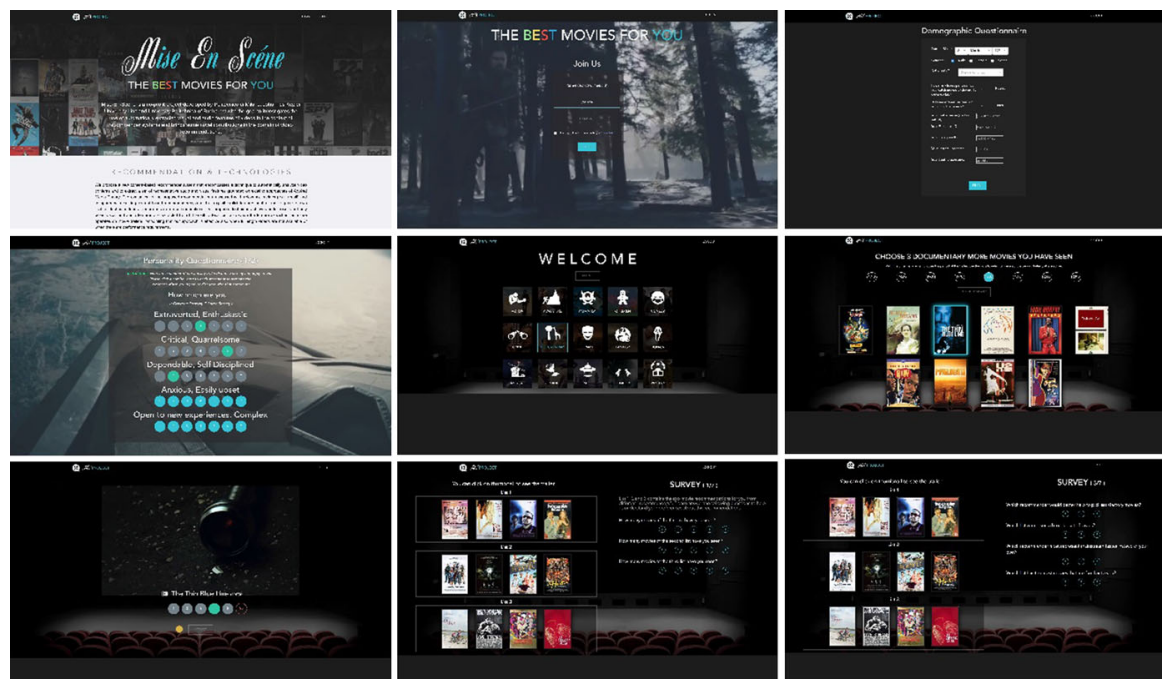

Fig. 6 Screenshots of the MISRec web application, designed for movie recommendation and empirical studies. The user needs to register, answer demographic and personality questionnaires, select his/her favorite genre, and rate some movies by looking at their trailers. Then, he/she is presented with 3 recommendation lists and a list of questions about perceived quality

process, where each participant (user) is asked to specify his/her e-mail address, user name, and password (see Fig. 6 top-middle). For users who wish to remain anonymous, we provide the option to conceal their true email address. Afterwards, the user is asked to provide basic demographics (age, gender, education, nationality, and number of movies watched per month, consumption channels, some optional social media IDs, such as Facebook, Twitter, and Instagram). After the user has registered for the system and provided his/her basic demographic information, he/she is asked to fill out the Ten-Item Personality Inventory (TIPI) questionnaire (see Fig. 6 middle-left) so that the system can assess his/her Big Five personality traits (openness, conscientiousness, extroversion, agreeableness, and neuroticism) (McCrae and John 1992). Then, for preference elicitation (Chen and $\mathrm{Pu} 2004$ ), the user is invited to browse the movie catalog from his/her favorite genre and to scroll through productions from different years in a user-friendly manner (see Fig. 6 center and middle-right). The user initially selects four movies as his/her favorites.

The user can watch the trailers for the selected movies and provide ratings for them using a 5-level Likert scale ( $1=$ low interest in/appreciation for the movie to $5=$ high interest in/appreciation for the movie). The user can also report a movie (if the trailer is not correctly displayed) and the movie will be skipped (see Fig. 6 bottom-left). After that, on the basis of these ratings and the content features described in Sect. 3.1, three categories of recommendation lists are presented to the user: (i) audio-based recommendation using BLF or i-vectors as features, (ii) visual-based recommendation using AVF or Deep as features, (iii) metadata-based recommendation using genre or tag as features. In each of the three recommendation categories, the recommendations are created using one of the two recommendation approaches (e.g., BLF or i-vectors 
for (i), and so on), chosen randomly. Since watching trailers is a time-consuming process, we decided to show only four recommendations in each of the three lists.

It is important to note that since we do not wish to overload the user with too much information, we avoid presenting him/her with six recommendation lists using all of the features. This would be the case in a within-subject design, where each subject uses all variants of the factorial designs simultaneously, i.e., six recommendation approaches in this case. Instead, we decided to use a between-subject design, where factorial designs are randomized for a given subject. Since our final goal is to have the user compare the three recommendation classes (i.e., audio vs. visual vs. metadata) at the same time, the way we implemented the between-subject design randomizes each of the two instances of each category for a given user. Therefore, each user compares one out of eight possible combinations: (BLF, AVF, genre), (i-vector, AVF, genre), (BLF, AVF, tag), (i-vector, AVF, tag) and so forth. ${ }^{20}$ This gives us more flexibility in handling all this information and obtaining reliable responses. Finally, to avoid possible biases or learning effects, the positions of the recommendation lists are randomized for each user.

\subsection{Results}

In this section, we present the user-perceived accuracy, satisfaction, personalization, diversity, and novelty. Before analyzing the survey responses, we cleaned the data by removing users who did not complete the questionnaire. We also removed users who were too fast in giving answers (less than $15 \%$ of the median time of all users) since we do not consider these users reliable. As the results of these filtering steps, 21 users are filtered out. Furthermore, users were asked to specify how many of the movies in each recommendation list they have seen. A list is included in the analysis only if the user has seen at least one movie from it. For example, if a user chooses a list as the recommendation most accurately matching his/her taste but has previously specified that he/she has not seen any movie from that list, we discard that list from his/her responses.

We compute a score for each recommender/feature with respect to the five performance measures. When recommendation lists are presented to the user, he/she has to choose one list out of the three as an answer to each question (cf. Table 8). Each selected list counts for a vote for the respective recommender that has created the list. Note that answers/scores given to questions marked with a + contribute positively to the final score, whereas scores to questions marked with a - contribute negatively. Finally, all votes given to each recommender are summed along each dimension (performance measure) and expressed as percentages, i.e., the relative frequency with which each recommender has been selected as the best one. The final results for the five dimensions are presented in Table 9 and discussed below.

\footnotetext{
20 Note that we could not use tag as a feature in Study A since tags are user-generated content. In cold items, no interactions with users have occurred yet; therefore, no tag could have been provided as a feature. Tags could be obtained via cross-domain techniques, but those are a vast research area and outside the scope of this paper. Tags could also be obtained by manual/editorial tagging, but that would be time-consuming and expensive, and therefore not suitable for a high rate of new items, which is the scenario of main interest for this paper.
} 
Table 9 Results of the user study with respect to the five tested perceived quality criteria in a real movie recommender system

\begin{tabular}{lllllll}
\hline Feature name & Feature type & Relevance & Diversity & Understands me & Satisfaction & Novelty \\
\hline tag & Metadata & 0.2632 & 0.1625 & 0.3133 & 0.2514 & 0.0577 \\
genre & Metadata & 0.2526 & 0.2857 & 0.2410 & 0.2404 & 0.1538 \\
i-vector & Audio & 0.1263 & 0.1875 & 0.0361 & 0.1093 & 0.2115 \\
BLF & Audio & 0.0316 & 0.1250 & 0.0120 & 0.0601 & 0.0769 \\
deep & Visual & 0.2421 & 0.1750 & 0.3253 & 0.2459 & 0.1923 \\
AVF & Visual & 0.0842 & 0.0625 & 0.0723 & 0.0929 & 0.3077 \\
\hline
\end{tabular}

Perceived accuracy/relevance The following algorithms are perceived as the most accurate (relevant) by the subjects: tag, genre, and the SoA visual deep feature, with $26 \%, 25 \%$, and $24 \%$ of the votes, respectively. User-generated tags are rich semantic descriptors and, as expected, the respective feature is evaluated the best by the subjects; however, the difference from genre and deep features remains very small $(1-2 \%)$. Meanwhile, the lowest performance is obtained by the traditional audio and visual features BLF and AVF with 3\% and 8\% of the votes, respectively. I-vector aggregates $13 \%$ of the votes. These results are in agreement with our expectations in that, as a standalone feature, the proposed SoA feature, deep, and i-vector show the most promising results compared with traditional multimedia features; e.g., Deep achieves a result of $24 \%$ in comparison with 8\% for AVF, which represents an improvement of about $300 \%$.

Understands me and satisfaction The results of users' perceived personalization (captured by the questions in the "Understands Me" category) and the overall feeling of the experience with the recommender system (captured by the questions in the "Satisfaction" category) show superior performance for Deep and tag features, with $32 \%$ and $31 \%$ of the votes, while genre is ranked lower, with $24 \%$ of the votes. For user satisfaction, the best performance is perceived for tag, deep, and genre features, with $25 \%, 24 \%$, and $24 \%$ of the user votes, respectively. The lowest performance is obtained by the traditional audio and visual features (between 7 and 10\%). We can also note that the results along the above perceived quality metrics are highly correlated (Pearson's correlation coefficient is 0.9735 ). The only exception is audio, in which we can find a difference in two dimensions between the performance obtained by SoA i-vectors (compare $3.6 \%$ vs. $11 \%$ ) and by traditional BLFs (compare $1.2 \%$ vs. $6 \%$ ). The results of "Understands me" and "Satisfaction" are also highly correlated with perceived accuracy (Pearson's correlation coefficients are 0.9390 and 0.9897 , respectively.). This can indicate that the users' perception of personalization and satisfaction is the same as accuracy and that users respond to the questions belonging to these categories in a similar way.

Diversity The results for the perceived diversity indicate that the best performance is achieved by genre (29\%) — substantially higher than i-vector, Deep, and tag, with $19 \%$, $18 \%$, and $16 \%$ of the votes, respectively. On the other hand, both traditional visual and audio features, AVF and BLF, show the lowest perceived diversity, attracting only $13 \%$ and $6 \%$ of the votes, respectively. The results for diversification are slightly different 
than those gained in our original user study (Deldjoo et al. 2018b) and show that users perceived recommendation by genre the most diverse (while perceived highly relevant too). Perhaps this is because users do not mentally compute list diversification based on genre diversity but also consider other attributes (e.g., the appearance of the DVD cover) when they are asked to indicate the most diversified recommendation list. Another reason could be that one of the questions explicitly asks for diversity of mood, and the same genre can have movies with very different moods (e.g., in sci-fi).

Novelty Results for novelty are surprising in several ways. Firstly, it is the traditional visual features, AVF, which have the highest amount of perceived novelty, gaining as much as $31 \%$ of votes, followed by the SoA audio and visual features i-vector and deep with $21 \%$ and $19 \%$ of the votes, respectively. Meanwhile, the tag feature has attracted a very small amount, i.e., only $5 \%$, of the scores for perceived novelty. Since tags are user-assigned, they have a high semantic content and capture something specific about the user perception of the movie. Therefore, similar tags may yield to recommendations not perceived as novel.

Globally, the results of our study on perceived recommendation quality indicate that perceived quality of recommendations is high for the SoA visual and audio features (Deep and i-vector) along most investigated performance measures. The exception is the user's perceived personalization ("Understands Me") for which i-vector performs poorly (but Deep visual performs best). For the remaining dimensions, these SoA features are ranked in the top 3 of all investigated features. Especially when it comes to novelty, SoA audio and visual features by far outperform metadata features. Overall, each feature has its merits, which again support our proposal for multimodal recommendation approaches.

\section{Conclusions and future perspectives}

In this work, we presented a framework for new movie recommendation by exploiting rich item descriptors and a novel recommendation model. We compared our system to some standard metadata-based methods that use genres and casts (editorial metadata). Specifically, the proposed system integrates multimedia aesthetic visual features and audio block-level features, as well as novel, state-of-the-art deep visual features and $i$-vector audio features, together with genre and cast features, all of which are referred to as the movie genome. For exploiting the complementary information of different modalities, we proposed CCA to fuse movie genome descriptors into shorter and stronger descriptors. Lastly, we presented a novel recommendation model that leverages a two-step approach named collaborative-filtering-enriched content-based filtering $(\mathrm{CFeCBF})$. It exploits the collaborative knowledge of warm items (videos with interactions) to weight content information for cold items (videos without interactions) and improve the ability to recommend cold videos, for which interactions and user-generated content are rare or unavailable. The proposed system represents a practical solution for alleviating the CS problem, in particular, the extreme CS new item problem, where newly added items lack any interaction and/or user-generated content. 


\subsection{Discussion of the results}

For evaluation, we conducted two empirical studies: (i) a system-centric study to measure the offline quality of recommendations in terms of accuracy (NDCG and MAP) and beyond accuracy (list diversity, distributional diversity, and item coverage) (cf. Sect. 4); (ii) a preliminary, user-centric online experiment to measure different subjective metrics, including relevance, satisfaction, and diversity (cf. Sect. 5). In both studies, we used a dataset of more than 4000 movie trailers, which makes our approach more versatile, because trailers are more readily available than full movies.

In the first study, visual and audio features generally outperform the metadata features with respect to the two tested accuracy measures, with an average growth factor of $32 \%$ along NDCG ( $\min 14 \%$ and $\max 53 \%$ ) and $23 \%$ along MAP (min $7 \%$ and max $42 \%$ ). The real improvement, however, is in the final system performance, in which the proposed system outperforms the baseline by a substantial margin of $80 \%$ along NDCG and $73 \%$ along MAP and also outperforms the simpler multimodal recommender model using CCA in a pure CBF system by $67 \%$ for NDCG and $68 \%$ for MAP. These results are promising and indicate the capability of our recommendation model to improve the utility of new item recommendation by leveraging rich $\mathrm{CF}$ data for existing warm items and utilizing them as feature weights to improve the content information in pure $\mathrm{CBF}$.

Moreover, in terms of beyond-accuracy measures, we can see that the genre-based recommender exhibits the lowest diversity, as could be expected. In addition, our results show that the multimodal recommender is able to provide substantially higher coverage and improved distributional diversity on all reported metrics. This means that a multimodal recommender is less prone to popularity bias; in particular, multimodal recommendations generated by our $\mathrm{CFeCBF}$ model show a significant improvement along (almost) all reported beyond-accuracy metrics, while not penalizing the accuracy and even improving it substantially.

When an item transition from cold to warm we can see that CF is able to outperform $\mathrm{CFeCBF}$ very soon in terms of accuracy metrics on a dataset with significant popularity bias, while CFeCBF still exhibit much better ability to leverage all the available items. The strength of the two algorithms may be combined allowing to exploit the superior recommendation quality of $\mathrm{CF}$ for warm items and the much greater coverage of $\mathrm{CFeCBF}$ to recommend cold items, whose low popularity renders the transition to warm slower.

In the user study, results show that the perceived recommendation for state-of-theart visual (Deep) and audio (i-vector) features are meaningful. With the exception of the user's perceived personalization, in which i-vector performed poorly, these audio and visual features are ranked in the top 3 of all investigated features. In some cases, such as for the perceived novelty, the improvement of these features over metadata was significantly high. Overall, the results of the user study show that each feature has advantages and supports our proposal for multimodal recommendation approaches. 


\subsection{Answers to research questions}

RQ1: Can the exploitation of movie genome describing rich item information as a whole, provide better recommendation quality compared with traditional approaches that use editorial metadata such as genre and cast in CS scenarios? As the experiments have shown, multimedia features can provide a good alternative to editorial metadata such as genre and cast in terms of both accuracy and beyond-accuracy measures. The use of multimedia features can allow to increase the recommendation quality in terms of accuracy while also improving the ability of the recommender to leverage the whole catalogue of items.

RQ2: Which visual and audio information better captures users' movie preferences in CS scenarios? The most important improvement for the accuracy metric was achieved by exploiting the state-of-the-art deep features for the visual modality but traditional block-level features for the audio modality.

RQ3: Could we leverage user interaction to enrich cold item information? We proved that it is possible to effectively leverage user interactions and enrich the item descriptors by learning a set of feature weights associated with the descriptors. This would result in improving the recommendation quality of cold items over current editorial baselines (genre and cast).

\subsection{Limitations}

Recommendation model The proposed recommender model has a few limitations. Firstly, since it leverages item features, the quality and noisiness of item features have an impact on the ability to learn good feature weights. If an item has too few features, the resulting recommendations will exhibit limited diversity and the weights might embed some popularity bias. This is visible in Table 4 for AVF + Genre, which, while having good recommendation quality, exhibits lower InterL diversity with respect to the other cases. On the other hand, if the number of features is too high, the number of collaborative similarities might not be enough to ensure good weighs are learned.

Secondly, as the model leverages a collaborative model, this feature weighting scheme will not be applicable to any scenario. If the user-item interactions are too few, it is well known that the collaborative model will perform poorly in comparison to a pure $\mathrm{CBF}$ recommender. If this is the case, the learned weights will be approximating a poor collaborative model and therefore the resulting recommendations will not improve. Even so, however, it may still be possible to leverage a collaborative model on a smaller and denser portion of the dataset to learn only some of the weights. This is an aspect that can be studied more in detail.

Thirdly, in the case of Boolean features, $\mathrm{CFeCBF}$ is sensitive to items with very sparse features due to the fact that it can learn weights only for features available for cold items. Feature sparsity has the dual effect of both increasing the probability of new items having many new features, previously unobserved, and reducing the degree of freedom of the model. 
Finally, in our previous study (Deldjoo et al. 2016d), we concluded concluded that trailers and movies share similar characteristics in the recommendation scenario. However, the dataset used in Deldjoo et al. (2016d) was rather small (167 full movies and corresponding trailers were used for comparison). Also, the number of visual features was limited (only five features, cf. Rasheed et al. 2005). Due to these restrictions, the generalizability of our findings in Deldjoo et al. (2016d) might be restricted. Nevertheless, we argue that using trailers instead of full-length movies serves as a good proxy and has several advantages: trailers are accessible, are sensibly shorter that the entire movie, and preserve the main idea of the movie since they are designed to trigger the viewers interest in watching the entire movie. Results in the paper at hand show that the performance recommendation system that exploits movie genome is better in comparison with editorial metadata (using genre or cast). We believe this can be seen as a breakthrough to demonstrate that they can effectively replace the full movies. Lastly, depending on the strength of the video descriptors with respect to the CF information, the items may transition from cold to warm after even a single interaction. In popularity biased datasets a premature switch from $\mathrm{CFeCBF}$ to $\mathrm{CF}$ may result in poor catalogue exploration and therefore limited overall recommender effectiveness. This effect can be minimized by adopting strategies to allow a gradual switch between the two allowing the less popular items more time to collect the interactions they need to become warm, while benefitting from the higher recommendation quality of a CF for warm items. The choice of an optimal point where to switch between $\mathrm{CFeCBF}$ and $\mathrm{CF}$ remains challenging.

User study limitations The reported user study results should be considered preliminary. In fact, given the relatively low number of participants, the results may not be statistically significant. Given the complexity of the questionnaire, which takes more than half an hour to complete, as well as due to the specificity of the movie dataset used, i.e., the movies tend to be classic ones not easily available to the younger generation, it is very difficult to find reliable users and motivate them to participate in the study, even when considering a paying platform such as crowdsourcing.

\subsection{Future perspectives}

We believe our proposed movie recommendation framework can pave the way for a new paradigm in new product recommendation by exploiting $\mathrm{CFeCBF}$ models built on top of rich item descriptors extracted from content. Examples of such products include fashion (images), music (audio), and tourism (both images and audio) and generic videos. As a related future research line, we would like to understand in what ways affective metadata (metadata that describe the user's emotions) can be used for CBF of videos/movies, similar to the research (Tkalčič et al. 2010) carried out for images.

Regarding the carried out user study, currently it involves 101 subjects. This is while according to Knijnenburg and Willemsen (2015), approximately 73 subjects are necessary in every configuration to ensure statistical significance of results (i.e., about 600 subjects in total). This is an important limitation of our current work, which we plan to overcome in the future by hiring a larger number of reliable subjects. Furthermore, 
we plan to validate the generalization power of our new movie recommender model on video datasets of a different nature, such as full-length movies, movie clips and user-generated videos. An initial attempt at the former was published in our work (Deldjoo et al. 2018b) and at the latter in Deldjoo et al. (2018a), whose authors plan to release a publicly available dataset of movie clips. Part of these data is used in the MediaEval 2018 task "Recommending Movies Using Content". ${ }^{21}$

Last but not least, a feature analysis will be conducted to better understand how movie genome features contribute to the success of the combined features as part of future work.

Acknowledgements Open access funding provided by Johannes Kepler University Linz. This work was supported by the Austrian Ministry for Transport, Innovation and Technology, and the Ministry of Science, Research and Economy, and the Province of Upper Austria in the frame of the COMET center SCCH. The work of Mihai Gabriel Constantin and Bogdan Ionescu was partially supported by the Ministry of Innovation and Research, UEFISCDI, project SPIA-VA, agreement 2SOL/2017, Grant PN-III-P2-2.1-SOL-2016-020002 .

Open Access This article is distributed under the terms of the Creative Commons Attribution 4.0 International License (http://creativecommons.org/licenses/by/4.0/), which permits unrestricted use, distribution, and reproduction in any medium, provided you give appropriate credit to the original author(s) and the source, provide a link to the Creative Commons license, and indicate if changes were made.

\section{References}

Adomavicius, G., Kwon, Y.: Improving aggregate recommendation diversity using ranking-based techniques. IEEE Trans. Knowl. Data Eng. 24(5), 896-911 (2012)

Adomavicius, G., Zhang, J.: Stability of recommendation algorithms. ACM Trans. Inf. Syst. (TOIS) 30(4), $23(2012)$

Aggarwal, C.C.: Content-based recommender systems. In: Recommender Systems, pp. 139-166. Springer, Berlin (2016a). https://dblp.uni-trier.de/rec/bibtex/books/sp/Aggarwal16

Aggarwal, C.C.: Evaluating recommender systems. In: Recommender Systems, pp. 225-254. Springer, Berlin (2016b). https://dblp.uni-trier.de/rec/bibtex/books/sp/Aggarwal16

Antenucci, S., Boglio, S., Chioso, E., Dervishaj, E., Shuwen, K., Scarlatti, T., Ferrari Dacrema, M.: Artistdriven layering and user's behaviour impact on recommendations in a playlist continuation scenario. In: Proceedings of the ACM Recommender Systems Challenge 2018 (RecSys Challenge '18) (2018)

Bartolini, I., Moscato, V., Pensa, R.G., Penta, A., Picariello, A., Sansone, C., Sapino, M.L.: Recommending multimedia objects in cultural heritage applications. In: International Conference on Image Analysis and Processing, pp. 257-267. Springer, Berlin (2013)

Bernardis, C., Ferrari Dacrema, M., Cremonesi, P.: A novel graph-based model for hybrid recommendations in cold-start scenarios. In: Proceedings of the Late-Breaking Results Track Part of the Twelfth ACM Conference on Recommender Systems. ACM (2018). arXiv:1808.10664

Bertin-Mahieux, T., Ellis, D.P., Whitman, B., Lamere, P.: The million song dataset. In: Proceedings of the 12th International Society for Music Information Retrieval Conference, pp. 591-596. Miami, USA (2011)

Bobadilla, J., Ortega, F., Hernando, A., Bernal, J.: A collaborative filtering approach to mitigate the new user cold start problem. Knowl. Based Syst. 26, 225-238 (2012)

Bordwell, D., Thompson, K., Smith, J.: Film Art: An Introduction, vol. 7. McGraw-Hill, New York (1997)

Bronstein, A.M., Bronstein, M.M., Kimmel, R.: The video genome. arXiv preprint arXiv:1003.5320 (2010)

Cella, L., Cereda, S., Quadrana, M., Cremonesi, P.: Deriving item features relevance from past user interactions. In: Proceedings of the 25th Conference on User Modeling, Adaptation and Personalization, pp. 275-279. ACM (2017)

21 http://www.multimediaeval.org/mediaeval2018/content4recsys. 
Chen, L., Pu, P.: Survey of preference elicitation methods. Technical report (2004)

Chu, W.T., Tsai, Y.L.: A hybrid recommendation system considering visual information for predicting favorite restaurants. World Wide Web 20(6), 1313-1331 (2017)

Cisco visual networking index: global mobile data traffic forecast update, 2015-2020 white paper. http://www.cisco.com/c/en/us/solutions/collateral/service-provider/visual-networking-indexvni/mobile-white-paper-c11-520862.html. Accessed: 1 Dec 2016

Cremonesi, P., Garzotto, F., Turrin, R.: Investigating the persuasion potential of recommender systems from a quality perspective: an empirical study. ACM Trans. Interact. Intell. Syst. (TiiS) 2(2), 11 (2012)

Cremonesi, P., Elahi, M., Deldjoo, Y.: Enhanced content-based multimedia recommendation method (2018). US Patent App. 15/277,490

Datta, R., Joshi, D., Li, J., Wang, J.Z.: Studying aesthetics in photographic images using a computational approach. In: European Conference on Computer Vision, pp. 288-301. Springer, Berlin (2006)

Daubechies, I.: Ten Lectures on Wavelets. Society for Industrial and Applied Mathematics, Philadelphia (1992)

de Gemmis, M., Lops, P., Musto, C., Narducci, F., Semeraro, G.: Semantics-aware content-based recommender systems. In: Recommender Systems Handbook, pp. 119-159. Springer, Berlin (2015). https:// dblp.uni-trier.de/rec/bibtex/reference/sp/2015rsh

Deldjoo, Y., Elahi, M., Quadrana, M., Cremonesi, P., Garzotto, F.: Toward effective movie recommendations based on mise-en-scène film styles. In: Proceedings of the 11th Biannual Conference on Italian SIGCHI Chapter, pp. 162-165. ACM (2015a)

Deldjoo, Y., Elahi, M., Quadrana, M., Cremonesi, P.: Toward building a content-based video recommendation system based on low-level features. In: International Conference on Electronic Commerce and Web Technologies, pp. 45-56. Springer, Berlin (2015b)

Deldjoo, Y., Elahi, M., Cremonesi, P., Garzotto, F., Piazzolla, P.: Recommending movies based on miseen-scene design. In: Proceedings of the 2016 CHI Conference Extended Abstracts on Human Factors in Computing Systems, pp. 1540-1547. ACM (2016a)

Deldjoo, Y., Elahi, M., Cremonesi, P., Moghaddam, F.B., Caielli, A.L.E.: How to combine visual features with tags to improve movie recommendation accuracy? In: International Conference on Electronic Commerce and Web Technologies, pp. 34-45. Springer, Berlin (2016b)

Deldjoo, Y., Elahi, M., Cremonesi, P.: Using visual features and latent factors for movie recommendation. In: CEUR-WS (2016c)

Deldjoo, Y., Elahi, M., Cremonesi, P., Garzotto, F., Piazzolla, P., Quadrana, M.: Content-based video recommendation system based on stylistic visual features. J. Data Semant. 5(2), 99-113 (2016d)

Deldjoo, Y., Cremonesi, P., Schedl, M., Quadrana, M.: The effect of different video summarization models on the quality of video recommendation based on low-level visual features. In: Proceedings of the 15th International Workshop on Content-Based Multimedia Indexing, p. 20. ACM (2017a)

Deldjoo, Y., Frà, C., Valla, M., Paladini, A., Anghileri, D., Tuncil, M.A., Garzotta, F., Cremonesi, P., et al.: Enhancing childrens experience with recommendation systems. In: Workshop on Children and Recommender Systems (KidRec'17)-11th ACM Conference of Recommender Systems (2017b)

Deldjoo, Y., Constantin, M.G., Dritsas, T., Schedl, M., Ionescu, B.: The mediaeval 2018 movie recommendation task: recommending movies using content. In: MediaEval 2018 Workshop (2018a)

Deldjoo, Y., Constantin, M.G., Eghbal-Zadeh, H., Schedl, M., Ionescu, B., Cremonesi, P.: Audiovisual encoding of multimedia content to enhance movie recommendations. In: Proceedings of the Twelfth ACM Conference on Recommender Systems. ACM (2018b). https://doi.org/10.1145/ 3240323.3240407

Deldjoo, Y., Constantin, M.G., Ionescu, B., Schedl, M., Cremonesi, P.: Mmtf-14k: A multifaceted movie trailer dataset for recommendation and retrieval. In: Proceedings of the 9th ACM Multimedia Systems Conference, MMSys, pp. 450-455 (2018c)

Deldjoo, Y., Elahi, M., Quadrana, M., Cremonesi, P.: Using visual features based on MPEG-7 and deep learning for movie recommendation. Int. J. Multimed. Inf. Retr. 7(4), 207-219 (2018d). https://doi. org/10.1007/s13735-018-0155-1

Deldjoo, Y., Schedl, M., Cremonesi, P., Pasi, G.: Content-based multimedia recommendation systems: definition and application domains. In: Proceedings of the 9th Italian Information Retrieval Workshop (IIR 2018). Rome, Italy (2018e)

Deldjoo, Y., Schedl, M., Hidasi, B., Kness, P.: Multimedia recommender systems. In: Proceedings of the 12th ACM Conference on Recommender Systems. ACM (2018f). https://doi.org/10.1145/3240323. 3241620 
Eghbal-Zadeh, H., Schedl, M., Widmer, G.: Timbral modeling for music artist recognition using i-vectors. In: 2015 23rd European Signal Processing Conference (EUSIPCO), pp. 1286-1290. IEEE (2015)

Eghbal-Zadeh, H., Lehner, B., Dorfer, M., Widmer, G.: CP-JKU submissions for DCASE-2016: a hybrid approach using binaural i-vectors and deep CNNs. Technical report, DCASE2016 Challenge (2016)

Ekstrand, M.D., Harper, F.M., Willemsen, M.C., Konstan, J.A.: User perception of differences in recommender algorithms. In: Proceedings of the 8th ACM Conference on Recommender Systems, RecSys '14, pp. 161-168. ACM, New York (2014). https://doi.org/10.1145/2645710.2645737

Elahi, M., Deldjoo, Y., Bakhshandegan Moghaddam, F., Cella, L., Cereda, S., Cremonesi, P.: Exploring the semantic gap for movie recommendations. In: Proceedings of the Eleventh ACM Conference on Recommender Systems, pp. 326-330. ACM (2017)

Elahi, M., Braunhofer, M., Gurbanov, T., Ricci, F.: User Preference Elicitation, Rating Sparsity and Cold Start, Chapter 8, pp. 253-294 (2018). https://doi.org/10.1142/9789813275355_0008

Elbadrawy, A., Karypis, G.: User-specific feature-based similarity models for top- $n$ recommendation of new items. ACM Trans. Intell. Syst. Technol. 6(3), 1-33 (2015). https://doi.org/10.1145/2700495

Ellis, D.P.: Classifying music audio with timbral and chroma features. ISMIR 7, 339-340 (2007)

Fatemi, N., Mulhem, P.: A conceptual graph approach for video data representation and retrieval. In: International Symposium on Intelligent Data Analysis, pp. 525-536. Springer, Berlin (1999)

Ferrari Dacrema, M., Gasparin, A., Cremonesi, P.: Deriving item features relevance from collaborative domain knowledge. In: Proceedings of KaRS 2018 Workshop on Knowledge-Aware and Conversational Recommender Systems. ACM (2018)

Gantner, Z., Drumond, L., Freudenthaler, C., Rendle, S., Schmidt-Thieme, L.: Learning attribute-to-feature mappings for cold-start recommendations. In: 2010 IEEE 10th International Conference on Data Mining (ICDM), pp. 176-185. IEEE (2010)

Haas, A.F., Guibert, M., Foerschner, A., Calhoun, S., George, E., Hatay, M., Dinsdale, E., Sandin, S.A., Smith, J.E., Vermeij, M.J., et al.: Can we measure beauty? Computational evaluation of coral reef aesthetics. PeerJ 3, e1390 (2015)

Haghighat, M., Abdel-Mottaleb, M., Alhalabi, W.: Fully automatic face normalization and single sample face recognition in unconstrained environments. Expert Syst. Appl. 47, $23-34$ (2016)

Harper, F.M., Konstan, J.A.: The movielens datasets: history and context. ACM Trans. Interact. Intell. Syst. (TiiS) 5(4), 19 (2016)

Herlocker, J.L., Konstan, J.A., Terveen, L.G., Riedl, J.T.: Evaluating collaborative filtering recommender systems. ACM Trans. Inf. Syst. 22(1), 5-53 (2004). https://doi.org/10.1145/963770.963772

Hinton, G.E., Srivastava, N., Krizhevsky, A., Sutskever, I., Salakhutdinov, R.R.: Improving neural networks by preventing co-adaptation of feature detectors. arXiv preprint arXiv:1207.0580 (2012)

Jalili, V., Matteucci, M., Goecks, J., Deldjoo, Y., Ceri, S.: Next generation indexing for genomic intervals. IEEE Trans. Knowl. Data Eng. (2018). https://doi.org/10.1109/TKDE.2018.2871031

Järvelin, K., Kekäläinen, J.: Cumulated gain-based evaluation of IR techniques. ACM Trans. Inf. Syst. 20(4), 422-446 (2002). https://doi.org/10.1145/582415.582418

Jégou, H., Douze, M., Schmid, C., Pérez, P.: Aggregating local descriptors into a compact image representation. In: 2010 IEEE Conference on Computer Vision and Pattern Recognition (CVPR), pp. 3304-3311. IEEE (2010)

Kaminskas, M., Bridge, D.: Diversity, serendipity, novelty, and coverage: a survey and empirical analysis of beyond-accuracy objectives in recommender systems. ACM Trans. Interact. Intell. Syst. 7(1), 2:1-2:42 (2016). https://doi.org/10.1145/2926720

Ke, Y., Tang, X., Jing, F.: The design of high-level features for photo quality assessment. In: 2006 IEEE Computer Society Conference on Computer Vision and Pattern Recognition, vol. 1, pp. 419-426. IEEE (2006)

Kelly, J.P., Bridge, D.: Enhancing the diversity of conversational collaborative recommendations: a comparison. Artif. Intell. Rev. 25(1), 79-95 (2006). https://doi.org/10.1007/s10462-007-9023-8

Kenny, P.: A small footprint i-vector extractor. Odyssey 2012, 1-6 (2012)

Kingma, D.P., Ba, J.: Adam: a method for stochastic optimization. arXiv preprint arXiv:1412.6980 (2014)

Knees, P., Schedl, M.: Music Similarity and Retrieval: An Introduction to Audio-and Web-Based Strategies, vol. 36. Springer, Berlin (2016)

Knijnenburg, B.P., Willemsen, M.C.: Evaluating recommender systems with user experiments. In: Recommender Systems Handbook, pp. 309-352. Springer, Berlin (2015). https://dblp.uni-trier.de/rec/bibtex/ reference/sp/2015rsh 
Knijnenburg, B.P., Willemsen, M.C., Gantner, Z., Soncu, H., Newell, C.: Explaining the user experience of recommender systems. User Model. User Adapt. Interact. 22(4-5), 441-504 (2012)

Koren, Y., Bell, R.: Advances in collaborative filtering. In: Ricci, F., Rokach, L., Shapira, B., Kantor, P.B. (eds.) Recommender Systems Handbook, pp. 77-118. Springer, Berlin (2015)

Krages, B.: Photography: The Art of Composition. Skyhorse Publishing Inc., New York (2012)

Krizhevsky, A., Sutskever, I., Hinton, G.E.: Imagenet classification with deep convolutional neural networks. In: Advances in Neural Information Processing Systems, pp. 1097-1105 (2012)

Lei, Y., Scheffer, N., Ferrer, L., McLaren, M.: A novel scheme for speaker recognition using a phoneticallyaware deep neural network. In: 2014 IEEE International Conference on Acoustics, Speech and Signal Processing (ICASSP), pp. 1695-1699. IEEE (2014)

Lew, M.S., Sebe, N., Djeraba, C., Jain, R.: Content-based multimedia information retrieval: state of the art and challenges. ACM Trans. Multimed. Comput. Commun. Appl. 2(1), 1-19 (2006). https://doi.org/ 10.1145/1126004.1126005

Li, C., Chen, T.: Aesthetic visual quality assessment of paintings. IEEE J. Sel. Top. Signal Process. 3(2), 236-252 (2009)

Li, Y., Hu, J., Zhai, C., Chen, Y.: Improving one-class collaborative filtering by incorporating rich user information. In: Proceedings of the 19th ACM International Conference on Information and Knowledge Management, pp. 959-968. ACM (2010)

Lika, B., Kolomvatsos, K., Hadjiefthymiades, S.: Facing the cold start problem in recommender systems. Expert Syst. Appl. 41(4), 2065-2073 (2014)

Liu, N.N., Yang, Q.: Eigenrank: a ranking-oriented approach to collaborative filtering. In: SIGIR '08: Proceedings of the 31st Annual International ACM SIGIR Conference on Research and Development in Information Retrieval, pp. 83-90. ACM, New York (2008). https://doi.org/10.1145/1390334.1390351

Liu, L., Chen, R., Wolf, L., Cohen-Or, D.: Optimizing photo composition. In: Computer Graphics Forum, vol. 29, pp. 469-478. Wiley, New York (2010). https://dblp.uni-trier.de/rec/bibtex/journals/ cgf/LiuCWC10

Liu, N.N., Meng, X., Liu, C., Yang, Q.: Wisdom of the better few: cold start recommendation via representative based rating elicitation. In: Proceedings of the fifth ACM Conference on Recommender Systems, pp. 37-44. ACM (2011)

Liu, J.H., Zhou, T., Zhang, Z.K., Yang, Z., Liu, C., Li, W.M.: Promoting cold-start items in recommender systems. PloS ONE 9(12), e113-457 (2014)

Logan, B.: Mel frequency cepstral coefficients for music modeling. In: Proceedings of the International Symposium on Music Information Retrieval (ISMIR). Plymouth, MA, USA (2000a)

Logan, B., et al.: Mel frequency cepstral coefficients for music modeling. In: ISMIR (2000b)

Lops, P., De Gemmis, M., Semeraro, G.: Content-based recommender systems: state of the art and trends. In: Ricci, F., Rokach, L., Shapira, B., Kantor, P. (eds.) Recommender Systems Handbook, pp. 73-105. Springer, Berlin (2011)

Ma, H., King, I., Lyu, M.R.: Learning to recommend with explicit and implicit social relations. ACM Trans. Intell. Syst. Technol. (TIST) 2(3), 29 (2011)

Matsuda, Y.: Color design. Asakura Shoten 2(4), 10 (1995)

McAuley, J., Targett, C., Shi, Q., Van Den Hengel, A.: Image-based recommendations on styles and substitutes. In: Proceedings of the 38th International ACM SIGIR Conference on Research and Development in Information Retrieval, pp. 43-52. ACM (2015)

McCrae, R.R., John, O.P.: An introduction to the five-factor model and its applications. J. Personal. 60(2), 175-215 (1992)

McFee, B., Barrington, L., Lanckriet, G.: Learning content similarity for music recommendation. IEEE Trans. Audio Speech Lang. Process. 20(8), 2207-2218 (2012)

Mei, T., Yang, B., Hua, X.S., Yang, L., Yang, S.Q., Li, S.: Videoreach: an online video recommendation system. In: Proceedings of the 30th Annual International ACM SIGIR Conference on Research and Development in Information Retrieval, pp. 767-768. ACM (2007)

Mei, T., Yang, B., Hua, X.S., Li, S.: Contextual video recommendation by multimodal relevance and user feedback. ACM Trans. Inf. Syst. (TOIS) 29(2), 10 (2011)

Ning, X., Karypis, G.: Slim: Sparse linear methods for top-n recommender systems. In: 2011 11th IEEE International Conference on Data Mining, pp. 497-506. IEEE (2011)

Obrador, P., Schmidt-Hackenberg, L., Oliver, N.: The role of image composition in image aesthetics. In: 2010 17th IEEE International Conference on Image Processing (ICIP), pp. 3185-3188. IEEE (2010) 
Park, S.T., Chu, W.: Pairwise preference regression for cold-start recommendation. In: Proceedings of the Third ACM Conference on Recommender Systems, RecSys '09, pp. 21-28. ACM, New York (2009). https://doi.org/10.1145/1639714.1639720

Paudel, B., Christoffel, F., Newell, C., Bernstein, A.: Updatable, accurate, diverse, and scalable recommendations for interactive applications. ACM Trans. Interact. Intell. Syst. (TiiS) 7(1), 1 (2017)

Pilászy, I., Tikk, D.: Recommending new movies: even a few ratings are more valuable than metadata. In: Proceedings of the Third ACM Conference on Recommender Systems, pp. 93-100. ACM (2009)

Rasheed, Z., Sheikh, Y., Shah, M.: On the use of computable features for film classification. IEEE Trans. Circuits Syst. Video Technol. 15(1), 52-64 (2005)

Rendle, S.: Factorization machines with libFM. ACM Trans. Intell. Syst. Technol. (TIST) 3(3), 57:1-57:22 (2012)

Ribeiro, M.T., Lacerda, A., Veloso, A., Ziviani, N.: Pareto-efficient hybridization for multi-objective recommender systems. In: Proceedings of the Sixth ACM Conference on Recommender Systems, RecSys '12, pp. 19-26. ACM, New York (2012). https://doi.org/10.1145/2365952.2365962

Saveski, M., Mantrach, A.: Item cold-start recommendations: learning local collective embeddings. In: Proceedings of the 8th ACM Conference on Recommender systems, pp. 89-96. ACM (2014)

Schedl, M., Knees, P., McFee, B., Bogdanov, D., Kaminskas, M.: Recommender Systems Handbook. Chap. Music Recommender Systems, 2nd edn. Springer, Berlin (2015)

Schedl, M., Zamani, H., Chen, C., Deldjoo, Y., Elahi, M.: Current challenges and visions in music recommender systems research. IJMIR 7(2), 95-116 (2018). https://doi.org/10.1007/s13735-018-01542

Schein, A.I., Popescul, A., Ungar, L.H., Pennock, D.M.: Methods and metrics for cold-start recommendations. In: Proceedings of the 25th Annual International ACM SIGIR Conference on Research and Development in Information Retrieval, pp. 253-260. ACM (2002)

Seyerlehner, K., Widmer, G., Schedl, M., Knees, P.: Automatic music tag classification based on block-level features. In: Proceedings of the 7th Sound and Music Computing Conference (SMC). Barcelona, Spain (2010)

Seyerlehner, K., Schedl, M., Knees, P., Sonnleitner, R.: A refined block-level feature set for classification, similarity and tag prediction. In: 7th Annual Music Information Retrieval Evaluation eXchange (MIREX 2011). Miami (2011)

Sharma, M., Zhou, J., Hu, J., Karypis, G.: Feature-based factorized bilinear similarity model for cold-start top-n item recommendation. In: Proceedings of the 2015 SIAM International Conference on Data Mining, pp. 190-198. SIAM (2015)

Sheskin, D.J.: Handbook of Parametric and Nonparametric Statistical Procedures. CRC Press, Cambridge (2003)

Singh, A.P., Gordon, G.J.: Relational learning via collective matrix factorization. In: Proceedings of the 14th ACM SIGKDD International Conference on Knowledge Discovery and Data Mining, pp. 650-658. ACM (2008)

Smyth, B., McClave, P.: Similarity versus diversity. In: Proceedings of the 4th International Conference on Case-Based Reasoning: Case-Based Reasoning Research and Development, ICCBR '01, pp. 347-361. Springer, London (2001). http://dl.acm.org/citation.cfm?id=646268.758890

Snoek, C.G., Worring, M., Smeulders, A.W.: Early versus late fusion in semantic video analysis. In: Proceedings of the 13th Annual ACM International Conference on Multimedia, pp. 399-402. ACM (2005)

Suh, J.W., Sadjadi, S.O., Liu, G., Hasan, T., Godin, K.W., Hansen, J.H.: Exploring hilbert envelope based acoustic features in i-vector speaker verification using ht-plda. In: Proceedings of NIST 2011 Speaker Recognition Evaluation Workshop (2011)

Tkalčič, M., Burnik, U., Košir, A.: Using affective parameters in a content-based recommender system for images. User Model. User Adapt. Interact. 20(4), 279-311 (2010)

Vall, A., Dorfer, M., Eghbal-zadeh, H., Schedl, M., Burjorjee, K., Widmer, G.: Feature-combination hybrid recommender systems for automated music playlist continuation. User Model. User Adapt. Interact. (2019). https://doi.org/10.1007/s11257-018-9215-8

van den Oord, A., Dieleman, S., Schrauwen, B.: Deep content-based music recommendation. In: C.J.C. Burges, L. Bottou, M. Welling, Z. Ghahramani, K.Q. Weinberger (eds.) Advances in Neural Information Processing Systems 26, pp. 2643-2651. Curran Associates, Inc. (2013). http://papers.nips.cc/ paper/5004-deep-content-based-music-recommendation.pdf

Vargas, S., Castells, P.: Rank and relevance in novelty and diversity metrics for recommender systems. In: Proceedings of the $5^{\text {th }}$ ACM Conference on Recommender Systems (RecSys). Chicago (2011) 
Victor, P., Cornelis, C., Teredesai, A.M., De Cock, M.: Whom should i trust? The impact of key figures on cold start recommendations. In: Proceedings of the 2008 ACM Symposium on Applied Computing, pp. 2014-2018. ACM (2008)

Weimer, M., Karatzoglou, A., Smola, A.: Adaptive collaborative filtering. In: RecSys '08: Proceedings of the 2008 ACM Conference on Recommender Systems, pp. 275-282. ACM, New York (2008). https:// doi.org/10.1145/1454008.1454050

Xu, Y., Monrose, F., Frahm, J.M., et al.: Caught red-handed: toward practical video-based subsequences matching in the presence of real-world transformations. In: 2017 IEEE Conference on Computer Vision and Pattern Recognition Workshops (CVPRW), pp. 1397-1406. IEEE (2017)

Yang, B., Mei, T., Hua, X.S., Yang, L., Yang, S.Q., Li, M.: Online video recommendation based on multimodal fusion and relevance feedback. In: Proceedings of the 6th ACM International Conference on Image and Video Retrieval, pp. 73-80. ACM (2007)

Yuan, J., Shalaby, W., Korayem, M., Lin, D., AlJadda, K., Luo, J.: Solving cold-start problem in large-scale recommendation engines: a deep learning approach. In: 2016 IEEE International Conference on Big Data (Big Data), pp. 1901-1910. IEEE (2016)

Zettl, H.: Sight, Sound, Motion: Applied Media Aesthetics. Cengage Learning, Boston (2013)

Zhang, Z.K., Liu, C., Zhang, Y.C., Zhou, T.: Solving the cold-start problem in recommender systems with social tags. EPL (Europhys. Lett.) 92(2), 28002 (2010)

Zhang, L., Agarwal, D., Chen, B.C.: Generalizing matrix factorization through flexible regression priors. In: Proceedings of the fifth ACM Conference on Recommender Systems, pp. 13-20. ACM (2011)

Zhang, X., Cheng, J., Qiu, S., Zhu, G., Lu, H.: Dualds: a dual discriminative rating elicitation framework for cold start recommendation. Knowl. Based Syst. 73, 161-172 (2015)

Zhou, T., Kuscsik, Z., Liu, J.G., Medo, M., Wakeling, J.R., Zhang, Y.C.: Solving the apparent diversityaccuracy dilemma of recommender systems. Proceedings of the National Academy of Sciences 107(10), 4511-4515 (2010)

Zhou, K., Yang, S.H., Zha, H.: Functional matrix factorizations for cold-start recommendation. In: Proceedings of the 34th International ACM SIGIR Conference on Research and Development in Information Retrieval, pp. 315-324. ACM (2011)

Ziegler, C.N., McNee, S.M., Konstan, J.A., Lausen, G.: Improving recommendation lists through topic diversification. In: Proceedings of the 14th International Conference on the World Wide Web, pp. 22-32. ACM (2005)

Publisher's Note Springer Nature remains neutral with regard to jurisdictional claims in published maps and institutional affiliations.

Yashar Deldjoo is a research assistant at the Information Retrieval Laboratory of University of Milano Bicocca, Italy. He obtained his Masters degree in Electrical Engineering from Chalmers University of Technology, Sweden in 2010. He earned his Ph.D. with Distinction in Computer Science from Politecnico di Milano, Italy in 2018. His main research interests include recommender systems, user modeling, multimedia recommendation and multimedia computing. Despite being a very young researcher, Yashar has (co-)authored quite many refereed articles at internationally recognized conferences and peer-reviewed journals (among others, published in ACM RecSys, MMSys, CHI, IEEE Transactions on Knowledge and Data Engineering, and User Modeling and User-Adapted Interaction, Journal of Data Semantics and Springer journal of multimedia information retrieval). He is also holder of a US Patent. Yashar has served as a PC member or reviewer for top-tier conferences including SIGIR, CIKM, AAAI, ACM MM, RecSys, ECML-PKDD, ECIR, MMSys, IDC, IPMU as well as top-tier journals, including Expert Systems with Applications and IEEE Access.

Maurizio Ferrari Dacrema is a Ph.D. candidate at the Department of Electronics, Information and Bioengineering (DEIB) at Politecnico di Milano, Italy, where he graduated in Computer Science and Engineering in 2016. His research is now focused on building hybrid recommender systems via feature weighting, as well as on offline model evaluation. He has been a member of a regular panel for Pilot 3 of the Horizon2020 TeSLa Project.

Mihai Gabriel Constantin graduated from the Faculty of Automatic Control and Computers, University Politehnica of Bucharest (UPB) and is currently a Ph.D. candidate with the Faculty of Electronics, 
Telecommunications and Information Technology and a researcher with the Multimedia Lab, CAMPUS Research Center, UPB, Romania. His research focused on the study of methods for analyzing the visual impact of multimedia data. He has authored over 11 scientific publications and was involved in several Romanian/EU funded research projects. He was a member of the organizing team for several conferences (e.g., IEEE/ACM CBMI 2016, ACM ICMR 2017) and benchmarking tasks (e.g., 2018 MediaEval Recommending Movies Using Content task).

Hamid Eghbal-zadeh is a research assistant at the Johannes Kepler University (JKU) Linz, Austria. He received his Masters degree in Computer Engineering from the Shiraz State University, Iran in 2012. From 2012 to 2014, he worked as a researcher in the area of speech processing at Ozyegin University in Istanbul, Turkey. In 2014, he joined the Institute of Computational Perception at the Johannes Kepler University of Linz. During the following years, he worked as a researcher on representation learning from signals and sequences. He is currently pursuing his $\mathrm{Ph} . \mathrm{D}$. and his focus of research is on learning from data with incomplete label information.

Stefano Cereda is a Ph.D. student at Politecnico di Milano, Italy, in the Department of Electronics, Information and Bioengineering, where he received his Masters degree in Computer Science and Engineering in 2017. After his Masters degree he worked as a researcher in the topic of recommender system. His research is now focused on automatic hyperparameter optimization for performance tuning.

Markus Schedl is associate professor at the Johannes Kepler University (JKU) Linz, Austria, affiliated with the Institute of Computational Perception. He graduated in Computer Science from the Vienna University of Technology and earned his Ph.D. in Computer Science from the JKU. His main research interests include web and social media mining, data analytics, information retrieval, recommender systems, multimedia, and music information research. Markus (co-)authored more than 200 refereed conference papers and journal articles (among others, published in ACM Multimedia, RecSys, ICMR, SIGIR, ECIR, ISMIR, WEB/WWW, IEEE Visualization; Journal of Machine Learning Research, ACM Transactions on Information Systems, IEEE Transactions on Affective Computing, IEEE Multimedia, User Modeling and UserAdapted Interaction, PLOS ONE). Furthermore, he is associate editor of the Springer International Journal of Multimedia Information Retrieval and the Transactions of the International Society for Music Information Retrieval. He serves on the program committee of top-tier conferences, including WWW, RecSys, ICMR, and ACM Multimedia, and as reviewer for top-tier journals, including ACM Computing Surveys, Elsevier Pattern Recognition Letters, IEEE Transactions on Multimedia, Journal of the Association for Information Science and Technology, and ACM Transactions on Intelligent Systems and Technology.

Bogdan lonescu coordinates the CAMPUS Research Center at University Politehnica of Bucharest (UPB). He holds a double Ph.D. degree in image/video processing from UPB and University of Savoie, France. $\mathrm{He}$ is currently a tenured Professor with ETTI-UPB. His main research interests cover: multimedia/video/ image processing and analysis, multimedia content-based retrieval and machine learning for multimedia. He has authored over 160 scientific publications. He serves/served as guest co-editor for Image and Vision Computing, Multimedia Tools and Applications, and International Journal of Computer Vision; conference committee chair for various conferences, e.g., ACM Multimedia 2013 (proceedings co-chair), ACM Multimedia 2019 (publicity chair), IEEE/ACM CBMI 2016 (general chair), ACM ICMR 2017 (general chair), CLEF 2021 (general chair); lead organizer/co-organizer for several benchmark campaigns: MediaEval Retrieving Diverse Social Images, Violent Scenes Detection, Affective Impact of Movies Task, Predicting Media Interestingness, and Predicting Media Memorability, ImageCLEF 2017-2019, ChaLearn ICPR Multimedia Information Processing for Personality and Social Networks Analysis Challenge. He contributed to over 21 Romanian/EU funded research and strategic programmes, as principal investigator or as part of the research team.

Paolo Cremonesi is professor of Recommender Systems at the Computer Science Department of Politecnico di Milano. Paolo is also the co-founder of Moviri and ContentWise, the first and most successful start-ups from Politecnico di Milano. Paolo holds an MSc in Aerospace Engineering and a Ph.D. in Computer Science. His research interests include high-performance computing and recommender systems. He has published almost 200 papers in refereed journals, conferences and book chapters, and holds 5 patents. 


\section{Affiliations}

Yashar Deldjoo ${ }^{1}$ (1) - Maurizio Ferrari Dacrema ${ }^{1}$ - Mihai Gabriel Constantin ${ }^{2}$. Hamid Eghbal-zadeh ${ }^{3}$. Stefano Cereda ${ }^{1}$. Markus Schedl ${ }^{3}$ (D) Bogdan Ionescu ${ }^{2}$. Paolo Cremonesi ${ }^{1}$

Yashar Deldjoo

yashar.deldjoo@polimi.it

Maurizio Ferrari Dacrema

maurizio.ferrari@polimi.it

Mihai Gabriel Constantin

mgconstantin@imag.pub.ro

Hamid Eghbal-zadeh

hamid.eghbal-zadeh@jku.at

Stefano Cereda

stefano.cereda@polimi.it

Bogdan Ionescu

bionescu@imag.pub.ro

Paolo Cremonesi

paolo.cremonesi@polimi.it

1 Politecnico di Milano, Milan, Italy

2 University Politehnica of Bucharest, Bucharest, Romania

3 Department of Computational Perception, Johannes Kepler University Linz, Linz, Austria 